\title{
Skeletal cryptococcosis from 1977 to 2013
}

\author{
Heng-Xing Zhou ${ }^{\dagger}, \mathrm{Lu}_{\mathrm{Lu}}{ }^{+}$, Tianci Chu, Tianyi Wang, Daigui Cao, Fuyuan Li, Guangzhi Ning and \\ Shiqing Feng*
}

Department of Orthopaedics, Tianjin Medical University General Hospital, Tianjin, China

\section{Edited by:}

Souha S. Kanj, American University of Beirut Medical Center, Lebanon

\section{Reviewed by:}

Saara Vainio, VU University Medical Center, Netherlands

Ronald Paul Rabinowitz, University of Maryland School of Medicine,

USA

\section{*Correspondence:}

Shiqing Feng, Department of

Orthopaedics, Tianjin Medical

University, General Hospital

NO. 154, Anshan Road, Tianjin

300052, China

e-mail: shiqing.feng@yahoo.com

${ }^{\dagger}$ These authors have contributed equally to this work.
Skeletal cryptococcosis, an aspect of disseminated cryptococcal disease or isolated skeletal cryptococcal infection, is a rare but treatable disease. However, limited information is available regarding its clinical features, treatment, and prognosis. This systematic review examined all cases published between April 1977 and May 2013 with regard to the factors associated with this disease, including patient sex, age, and epidemiological history; affected sites; clinical symptoms; underlying diseases; laboratory tests; radiological manifestations; and delays in diagnosis, treatment, follow-up assessments, and outcomes. We found that immune abnormality is a risk factor but does not predict mortality; these observations are due to recent Cryptococcus neoformans var gattii (CNVG) outbreaks (Chaturvedi and Chaturvedi, 2011). Dissemination was irrespective of immune status and required combination therapy, and dissemination carried a worse prognosis. Therefore, a database of skeletal cryptococcosis cases should be created.

Keywords: skeletal cryptococcosis, Cryptococcus neoformans, immune status, underlying disease, dissemination

\section{INTRODUCTION}

Cryptococcosis, formerly known as torulosis, European blastomycosis, or Busse-Buschke disease, is caused by Cryptococcus neoformans (C neoformans). This species was first isolated from peach juice by Sanfelice in 1894 (Mitchell and Perfect, 1995; Jain et al., 2013). Cryptococcus is a spherical-to-oval, encapsulated, yeast-like fungus that is widespread in spoiled milk, soil, and bird droppings, especially pigeon excreta. $C$ neoformans can be divided into Cryptococcus neoformans var neoformans (CNVN) and Cryptococcus neoformans var gattii (CNVG), both of which are pathogens in humans and animals. They were not considered different varieties until 1970, when CNVG was officially suggested as a new species based on mounting evidence discovered since the first CNVG report in 1896 (Speed and Dunt, 1995; Chaturvedi and Chaturvedi, 2011; Harris et al., 2011). In addition, an on-going CNVG outbreak originated in 1999 and reappeared in 2004 (Chaturvedi and Chaturvedi, 2011). The most affected organs are the lungs and central nervous system, but virtually any organ (e.g., the skin, joints, eyes, urinary tract, liver, prostate, myocardium, muscles, kidneys, and bone) can be involved through lymphangitic and hematogenous spread after inhaling fungal propagules. Skeletal cryptococcosis is rare. Furthermore, this disease can be divided into two types: skeletal cryptococcosis, which is an aspect of disseminated cryptococcosis, and primary skeletal cryptococcosis, which does not involve other tissues (Chleboun and Nade, 1977; Behrman et al., 1990; Wood and Miedzinski, 1996). Due to its low morbidity rate, little is known about this disease. Moreover, its basic clinical features, treatment, and prognosis have long perplexed clinicians. Serious consequences, including death, have occurred in certain patients (Singh and Xess, 2010). Therefore, a systematical retrospective analysis of skeletal cryptococcosis is crucial for understanding this disease. Unfortunately, however, almost all of the current studies regarding this disease have been presented as case reports (Ramkillawan et al., 2013; Zhou et al., 2013), which provides little and indirect insight for understanding of skeletal cryptococcosis. Thus, a systematic, retrospective analysis of all published cases of skeletal cryptococcosis reported between April 1977 and the present time was conducted to clarify its clinical features, treatments, and prognoses-all of which are critical issues for fully understanding this disease. Importantly, treatment and prognostic suggestions based on the analysis are provided.

\section{MATERIALS AND METHODS SEARCH STRATEGY AND SELECTION CRITERIA}

A systematic online search was performed for cases reported over a 36-year period from April 1977 to the present time using PubMed, Medline, EBSCO, SpringerLink, Ovid, Highwire, ProQuest, and Wiley InterScience. We applied the following algorithm in both the medical subject heading (MeSH) and the search field. The MeSH terms "case reports" and "review" were combined with "Cryptococcus," "Cryptococcus neoformans," "osteomyelitis," or "immunocompromised host," and these MeSH terms were exploded when appropriate. Search terms such as "cryptococcosis," "skeletal," "bone," "joints," "skull," "arthritis," "disseminated," or "immunocompetent" were also combined with the MeSH terms to increase the number of relevant articles retrieved. Google Scholar was also searched, and the citations in each article were reviewed to identify additional references that were not retrieved during the primary search. Language 
restrictions were not applied, and two researchers independently conducted all searches.

"Disseminated skeletal cryptococcosis" was defined as an infection that involves two or more non-contiguous bone sites or an infection that involves one bone site associated with extraskeletal sites; patients with soft tissue collections or abscesses adjacent to the involved bone were excluded (Wood and Miedzinski, 1996). "Classically immunodeficient conditions" were considered to include corticosteroid use, HIV infection, interleukin-2 deficiency, and T cell defects (Speed and Dunt, 1995; Yu et al., 2012). Patients with other underlying diseases that affect immune function, such as diabetes mellitus, tuberculosis and connective tissue disorders, were considered relatively immunocompromised. "Osteomyelitis" was defined based on a positive radiograph, bone scan, or histopathology (Harris et al., 2011). "Relapse" was defined as the recurrence of symptoms at the previous disease site and the rediscovery of viable cryptococci from a previously checked sterile body site after successful primary therapy (Perfect et al., 2010).

\section{DATA COLLECTION AND ANALYSIS}

The following information was retrospectively reviewed: patient sex, age, and epidemiological history; involved sites; clinical symptoms; underlying diseases; laboratory tests; radiological manifestations; and delays in diagnosis, treatment, follow-up assessment, and outcomes. Outcome was recorded as either response (i.e., resolution or improvement of all signs and symptoms, including microbiological and serological abnormalities and radiographic changes due to infection) or failure (i.e., deterioration of the patient's condition based on clinical features and radiographic abnormalities, ultimately resulting in death) (Kontoyiannis et al., 2001).

All cases were epidemiologically and clinically analyzed. The hosts were categorized as patients with immune abnormalities (including classically immunodeficient and relatively immunocompromised status) or as immunocompetent.

\section{STATISTICAL ANALYSES}

Statistical analyses were performed using IBM SPSS 18.0 (IBM Corporation, Armonk, NY, USA). All continuous data were expressed as means \pm standard deviations (means \pm SDs), and comparisons were performed using One-Way ANOVAs. Categorical variables were compared using the $\chi^{2}$-test. All tests were two-tailed, and $p<0.05$ were considered significant.

\section{RESULTS}

In total, 80 articles (including one written in Spanish [case 50] and 79 written in English) that described 89 patients were collected and analyzed retrospectively (Table 1). Case 25 recorded only the affected site (orbit) and treatment (amphotericin [AMB] and ketoconazole); thus, this case was removed from the analysis due to unknown immune status.

\section{SEX AND AGE}

Forty-four (of 88, 50.0\%) males and 44 (of 88, 50.0\%) females were included. Their ages ranged from 16 months to 84 years with a mean $( \pm S D)$ of 39.9 years $( \pm 19.6$; Figure 1). Relatively immunocompromised hosts $(n=31,48.8 \pm 17.9$ years $)$ were older than immunocompetent hosts $(n=32,33.9 \pm 19.7$ years; ANOVA, $p=0.002)$ and classically immunodeficient hosts $(n=$ $25,36.7 \pm 18.5$ years; ANOVA, $p=0.018$ ); however, classically immunodeficient hosts were approximately the same age as the immunocompetent hosts (ANOVA, $p=0.587$ ).

\section{EPIDEMIOLOGICAL HISTORIES}

Thirteen (of 88, 14.8\%) patients had epidemiological histories (Table 2). The epidemiological histories of the patients with immune abnormalities (seven of 56, 12.5\%) and those who were immunocompetent (six of 32, 18.8\%) did not significantly differ $\left(\chi^{2}\right.$-test, $\left.p=0.629\right)$.

\section{INVOLVED SITES}

Regarding bone and joint infections, see Figures 2, 3A,B. Multiple site infections did not differ among the classically immunodeficient (10 of $25,40.0 \%$ ), relatively immunocompromised (13 of $29,44.8 \%$ ), and immunocompetent groups (10 of $32,31.3 \%$; $\chi^{2}$-test, $\left.p=0.542\right)$. Extra-skeletal cryptococcosis was found in 34 patients (Figure 3C). The patients categorized as classically immunodeficient (16 of $25,64.0 \%$ ) were more likely to have extra-skeletal infections than were those categorized as relatively immunocompromised ( 10 of $31,32.3 \% ; \chi^{2}$-test, $p=0.018$ ) and immunocompetent (eight of $32,25.0 \% ; \chi^{2}$-test, $p=0.003$ ), whereas patients categorized as relatively immunocompromised or immunocompetent did not differ in this regard $\left(\chi^{2}\right.$-test; $p=0.524$ ). Excluding case 25, 42 (of 88, 47.7\%) patients had disseminated cryptococcosis. The patients categorized as classically immunodeficient (17 of $25,68.0 \%$ ) were more likely to have disseminated cryptococcosis than were those categorized as immunocompetent (11 of 32, 33.4\%; $\chi^{2}$-test; $p=$ $0.012)$. Dissemination among the patients categorized as classically immunodeficient did not differ from that among those categorized as relatively immunocompromised (14 of 31, 45.2\%; $\chi^{2}$-test; $\left.p=0.087\right)$, nor did the dissemination among the patients categorized as relatively immunocompromised differ from that among those categorized as immunocompetent $\left(\chi^{2}\right.$-test; $p=0.382$ ).

\section{CLINICAL SYMPTOMS}

The predominant complaints included soft tissue swelling and pain, the duration of which ranged from acute admission to 3 years. Of the evaluable 86 patients (excluding cases 25,41 , and 50 whose data were not available), fever was observed in 18 (20.9\%) patients, but body temperature measurements were only available in 12 of these patients $(66.7 \%)$ and ranged from $37.4^{\circ} \mathrm{C}$ to $39.2^{\circ} \mathrm{C}$ (mean temperature $\left.=38.35 \pm 0.61^{\circ} \mathrm{C}\right)$.

\section{UNDERLYING DISEASES}

Of the 88 patients (excluding case 25), 25 (28.4\%) were categorized as classically immunodeficient, $31(35.2 \%)$ were relatively immunocompromised, and $32(36.7 \%)$ were immunocompetent. Corticosteroid use (18 of $25,72.0 \%)$ was the most common cause of classically immunodeficient status. Diabetes mellitus (nine of $31,29.0 \%$ ) followed by tuberculosis (seven of $31,22.6 \%$ ) and connective tissue disorders (five of $31,16.1 \%$ ) were the most common causes of the relatively immunocompromised status. Of the 32 patients in the immunocompetent group, 22 (68.8\%) were 
InUn

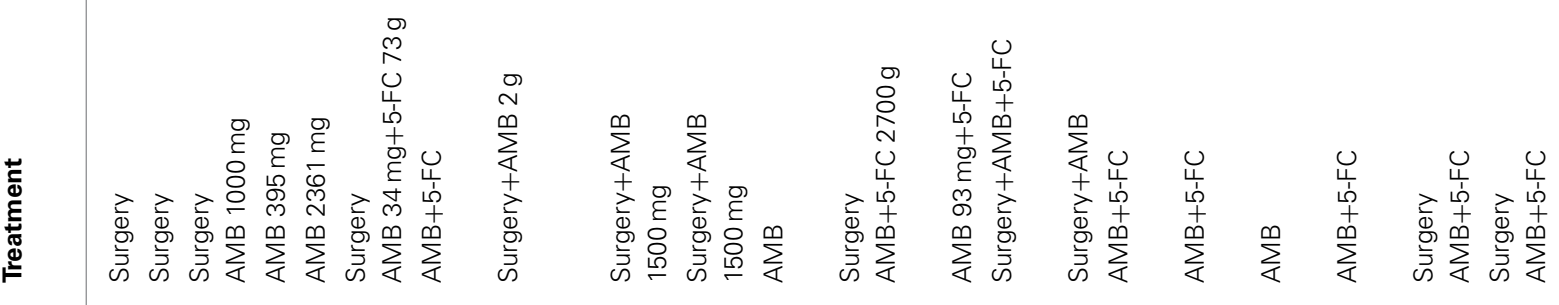
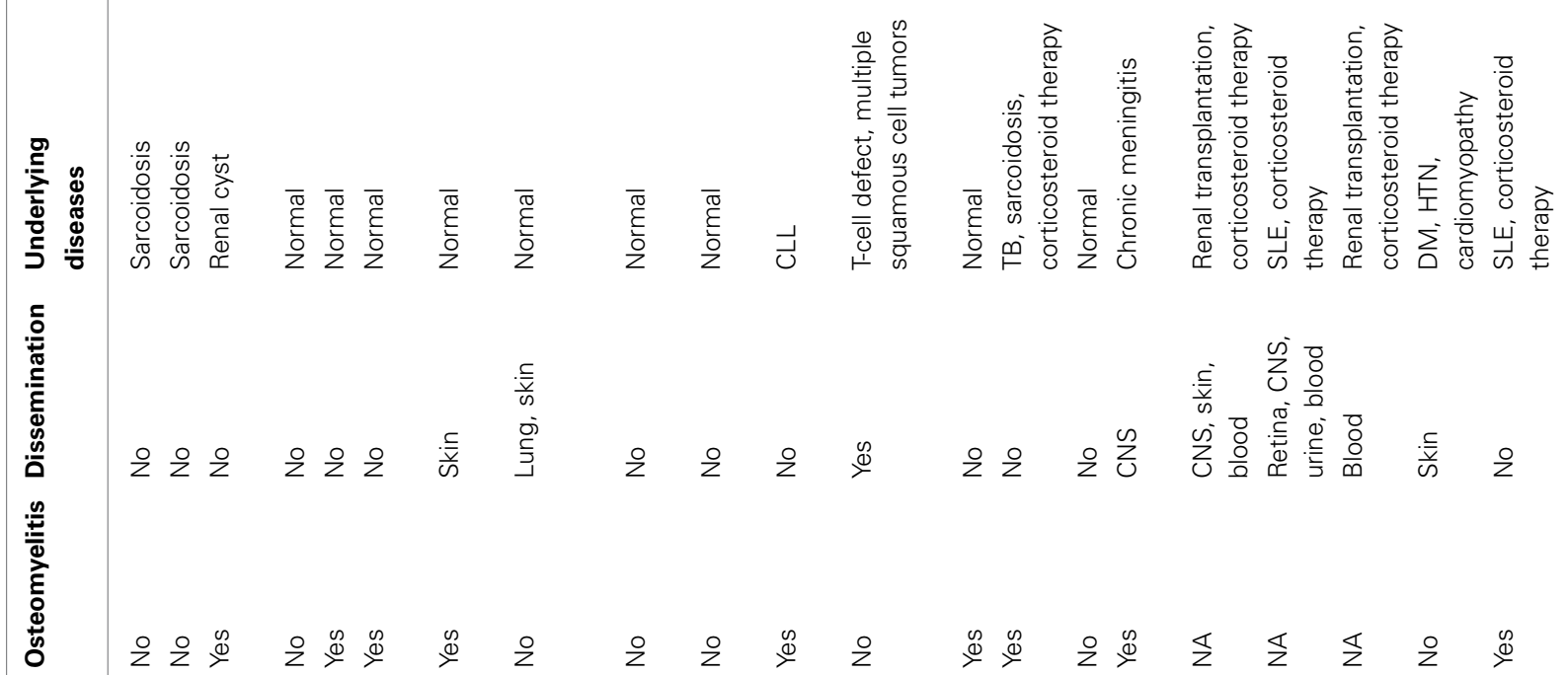

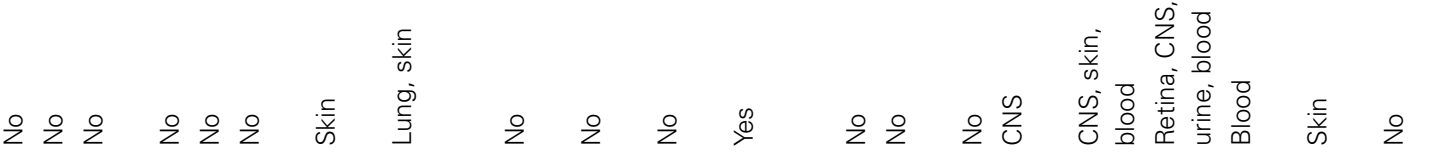

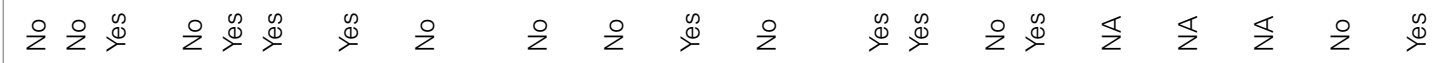

$\infty$ ๖

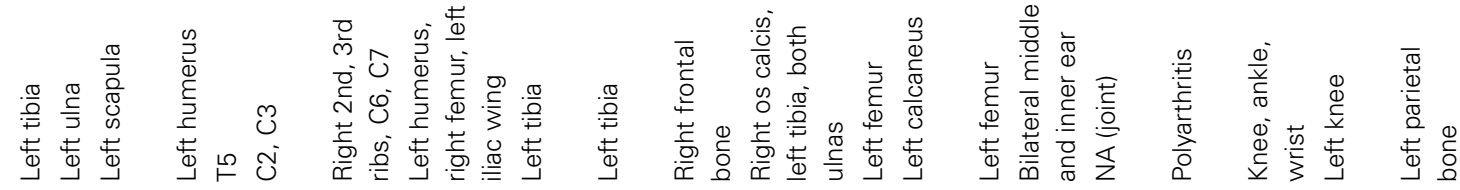

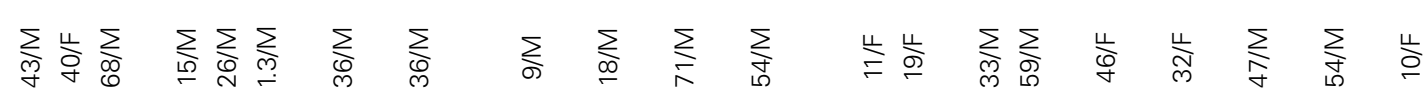
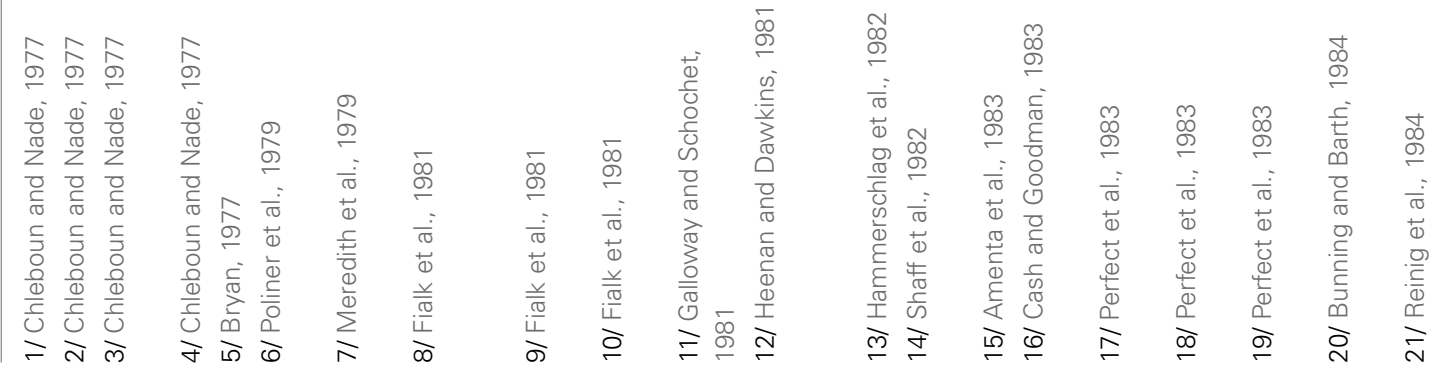


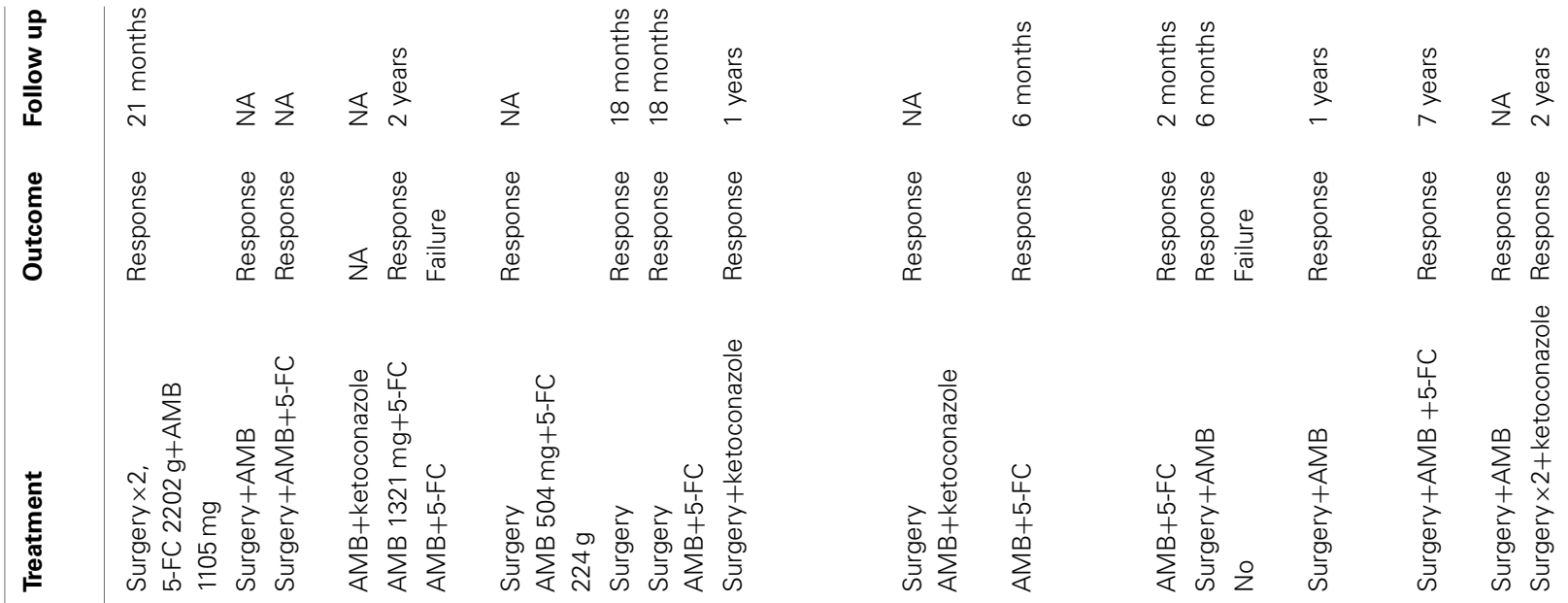

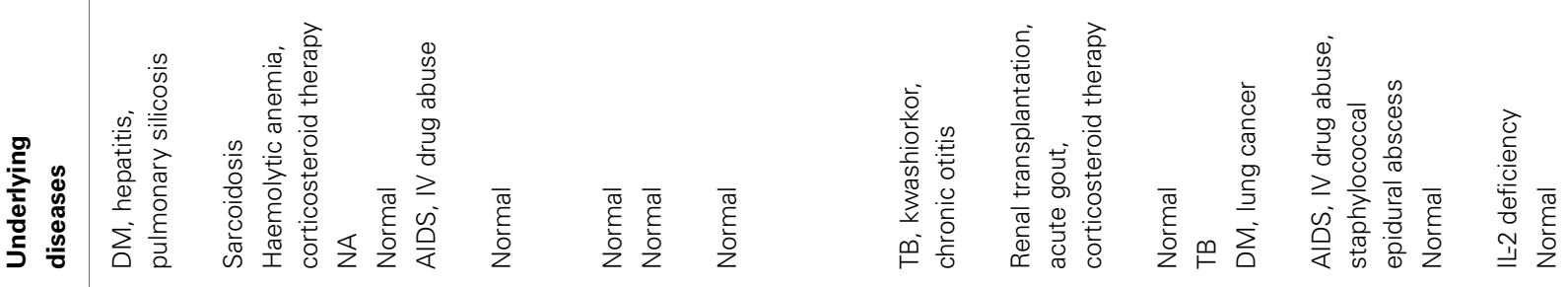

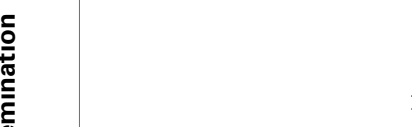

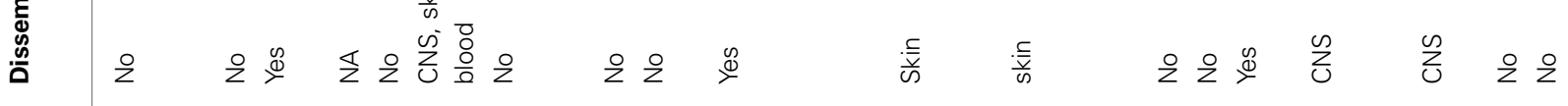
|

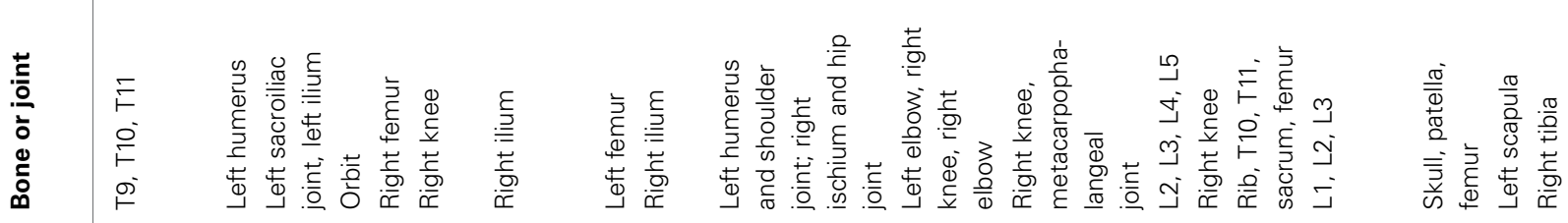

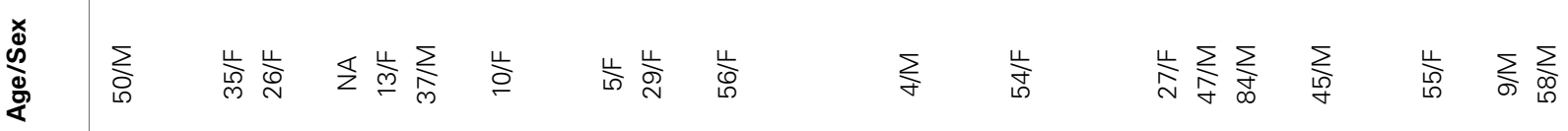

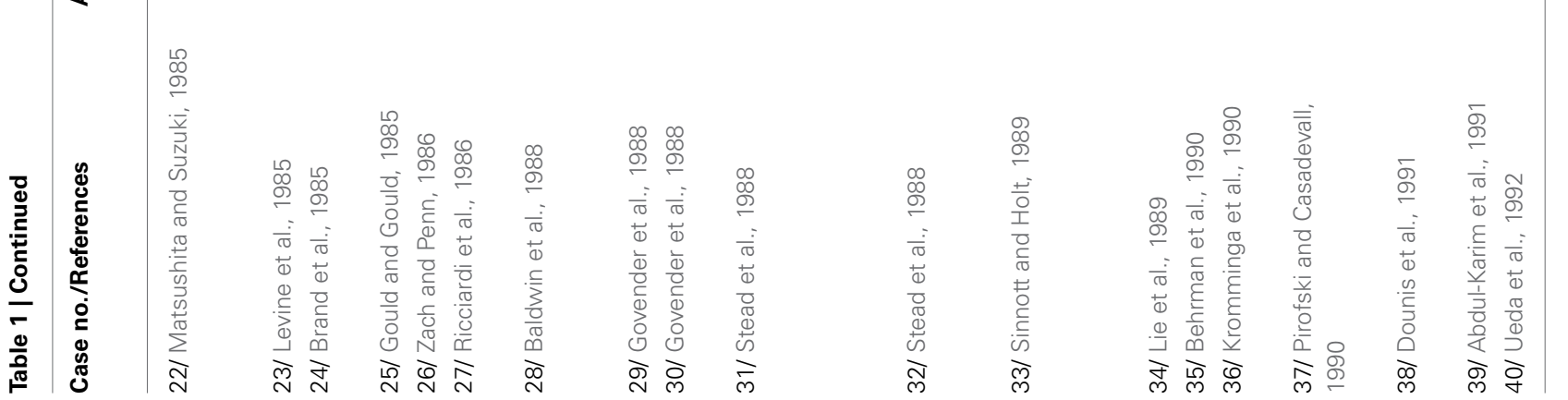




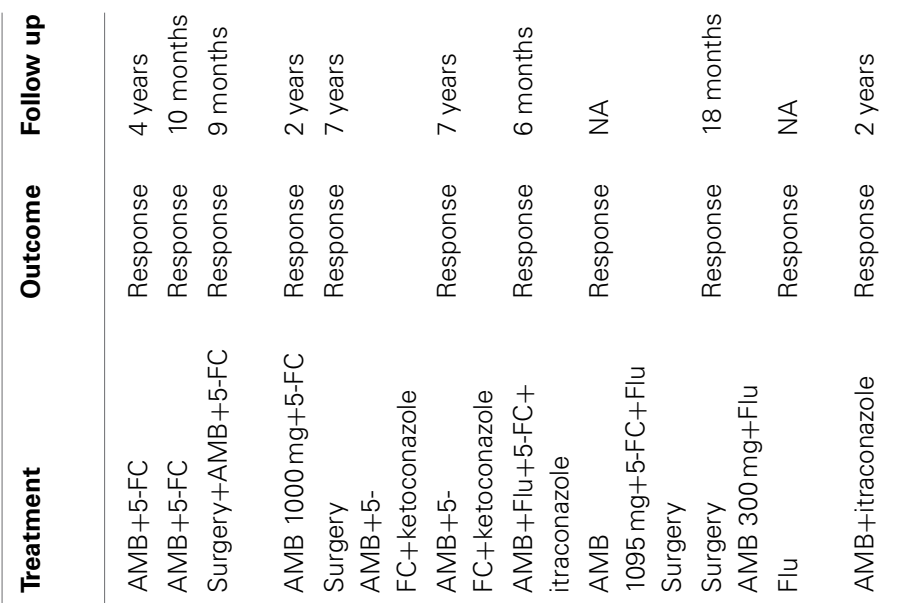

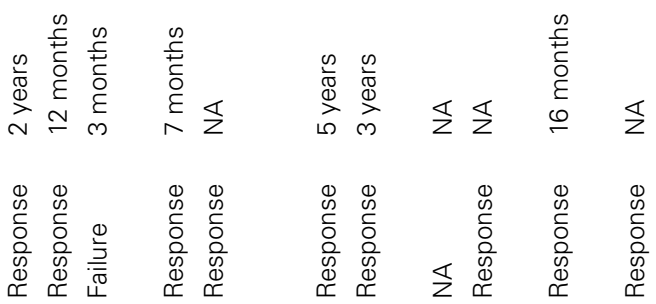

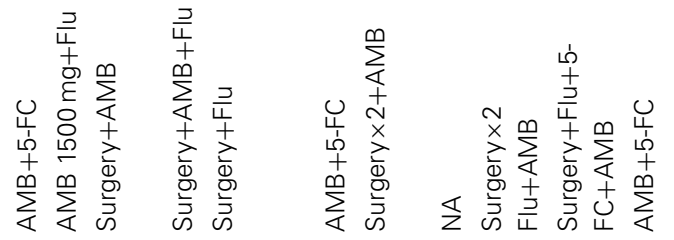
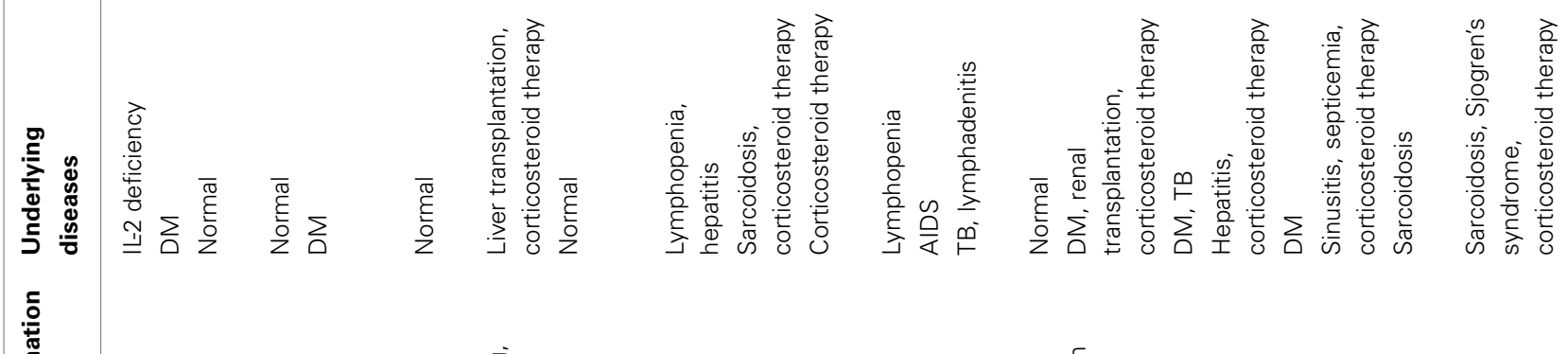

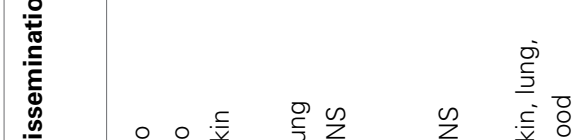

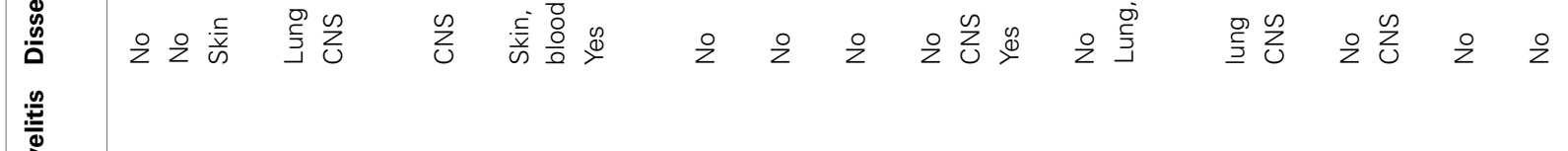

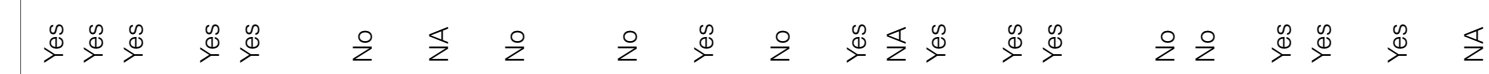

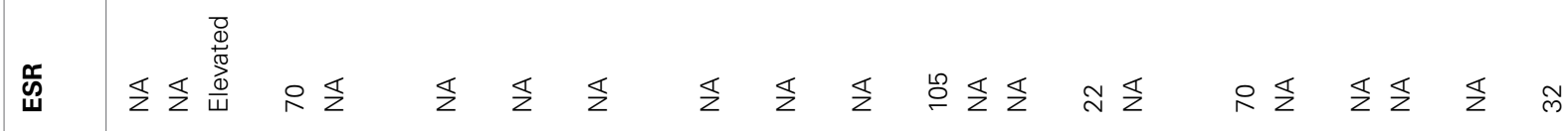

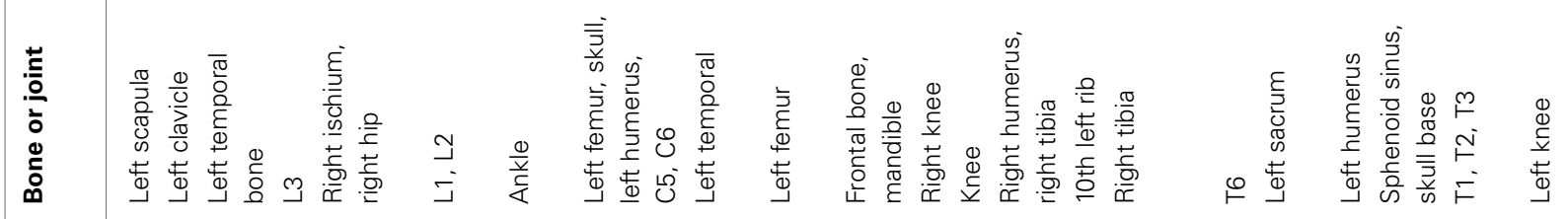

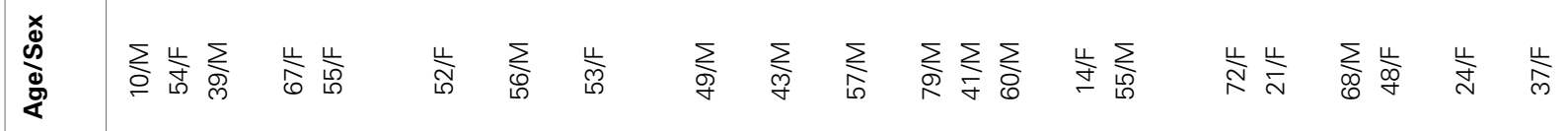
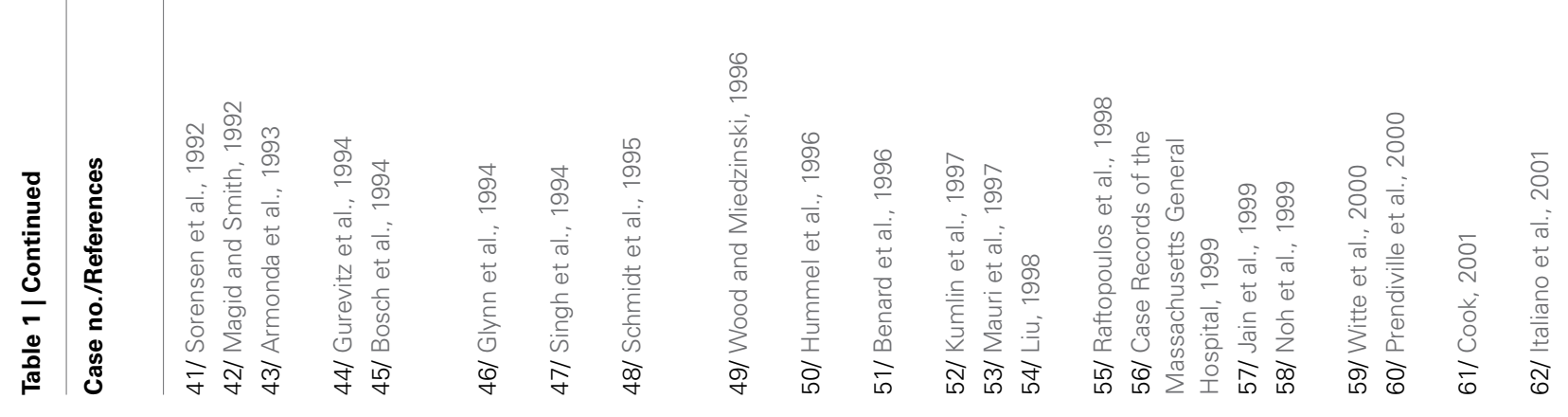


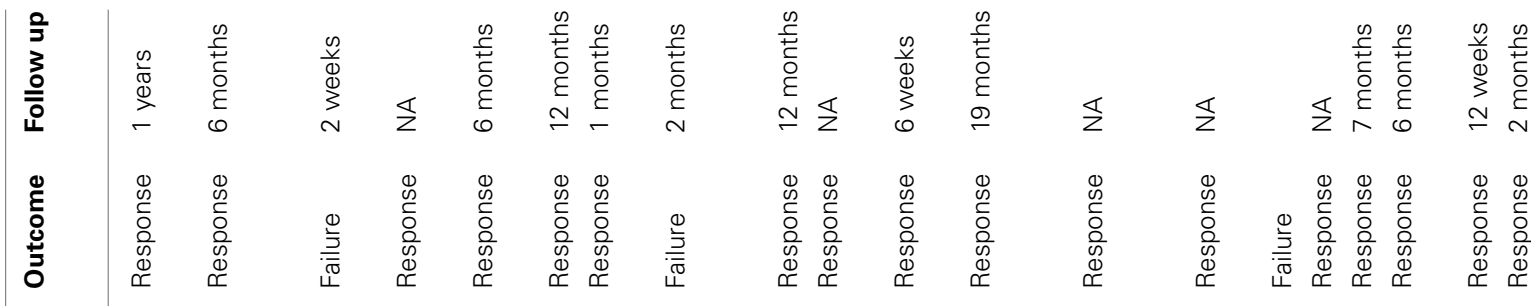

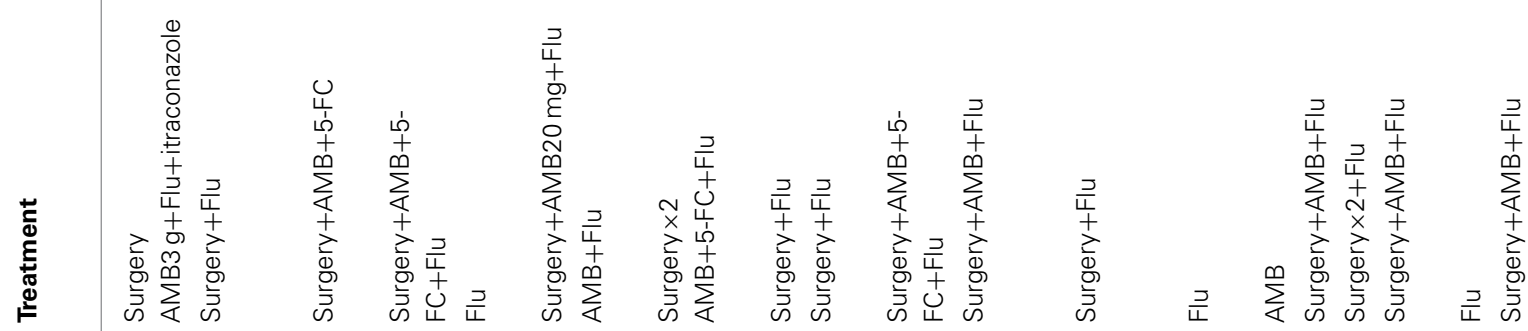

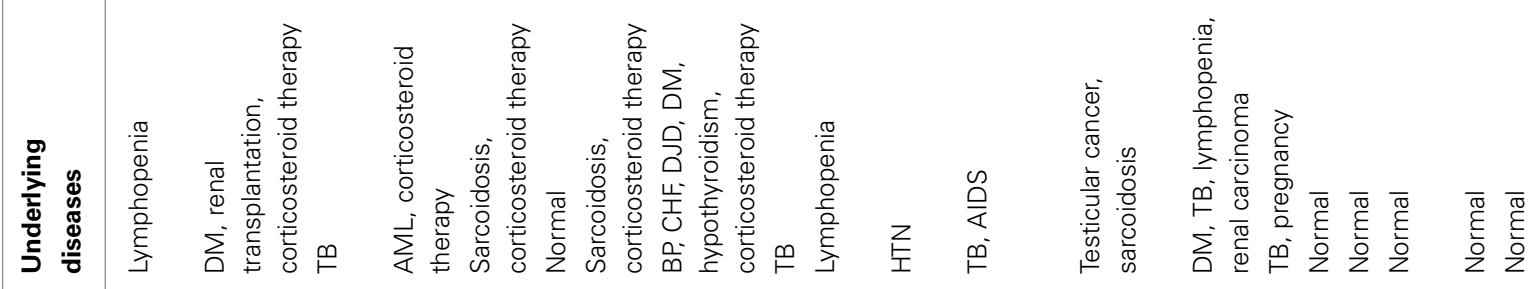

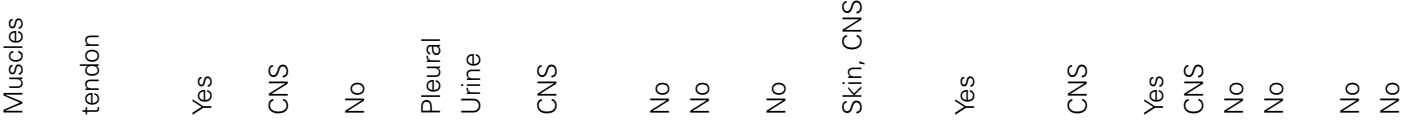

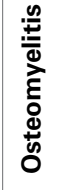

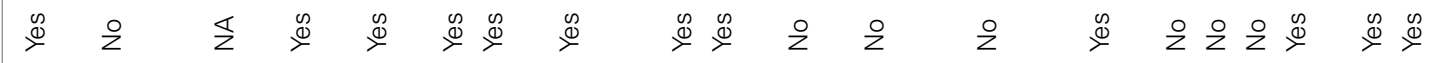

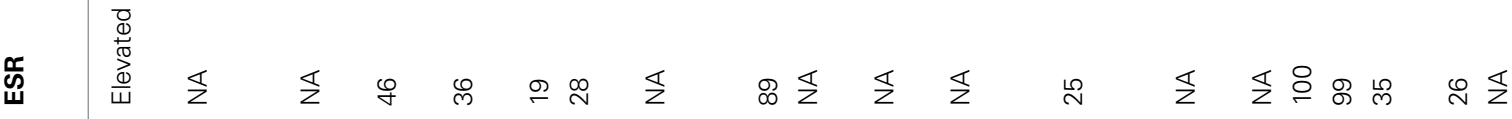

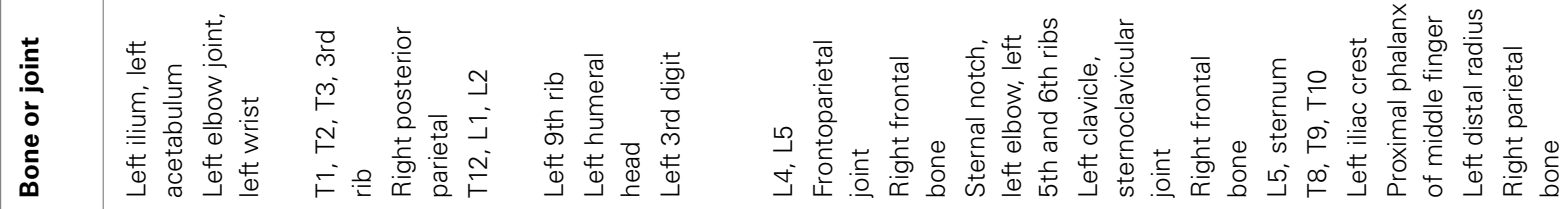
离

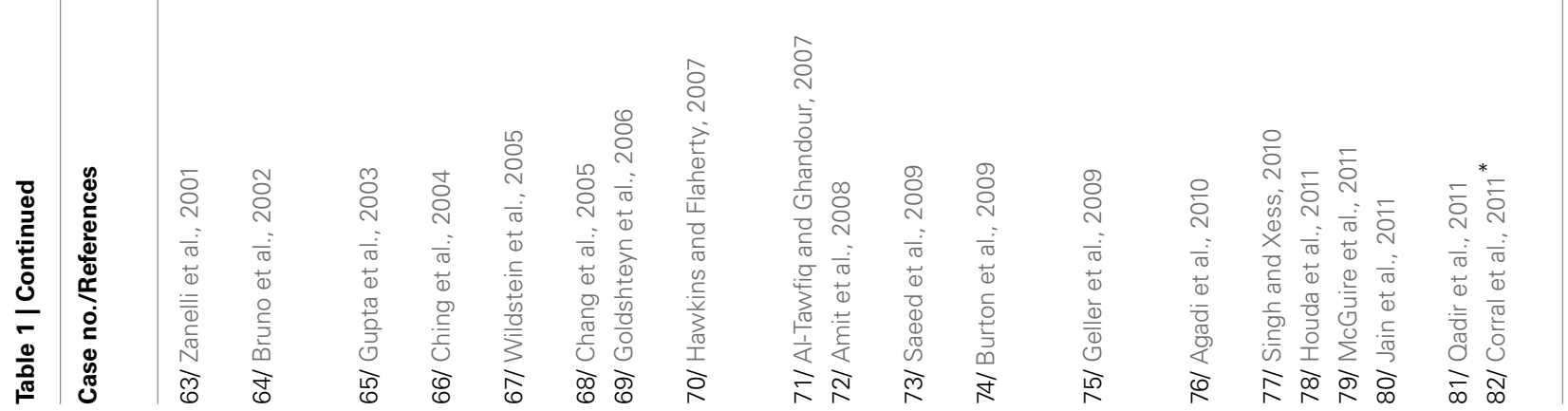




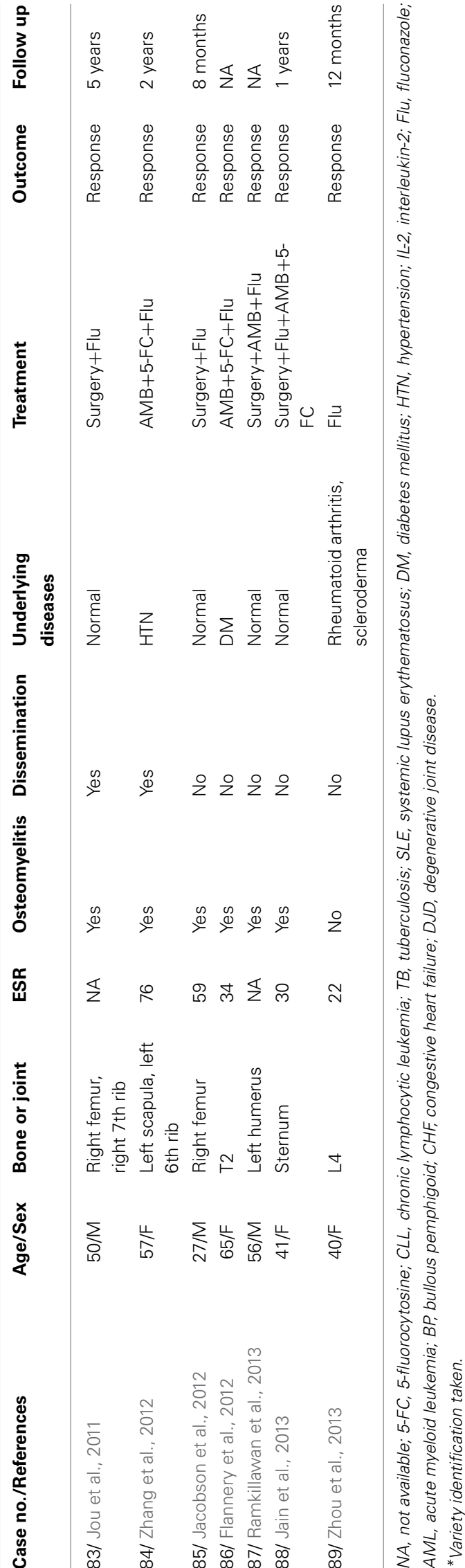

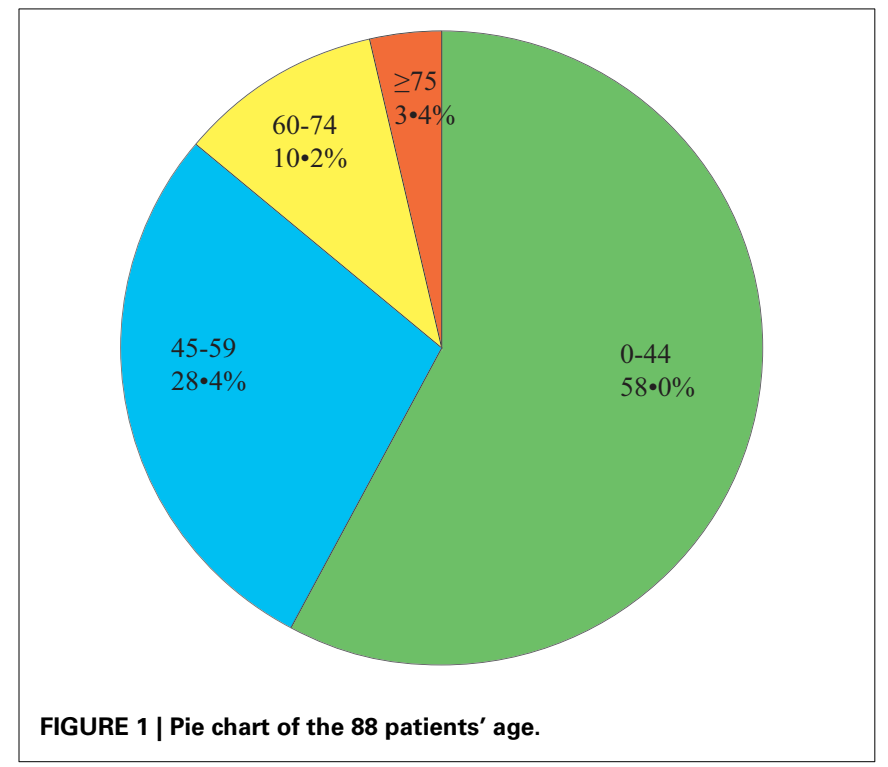

described in articles published before 1999, and 10 (31.2\%) were described in articles published after 2004. No immunocompetent patients were described between 1999 and 2004.

\section{LABORATORY TESTS}

The erythrocyte sedimentation rate (ESR) was documented for 40 (of 89, 44.9\%) patients. Thirty-seven (of 40, 92.5\%) ESRs were elevated (i.e., $>20 \mathrm{~mm} / \mathrm{h}$ for females and $>15 \mathrm{~mm} / \mathrm{h}$ for males) for 24 (of 37, 64.9\%) female patients and 13 (of 37, 35.1\%) male patients. Among the 24 female patients, definite elevated ESRs were documented in 23 (of 24, 95.8\%) patients (average, $52.0 \mathrm{~mm} / \mathrm{h} \pm 27.3$ ), whereas among the 13 male patients, definite elevated ESRs were documented in 10 (of 13, 76.9\%) patients (average, $49.0 \mathrm{~mm} / \mathrm{h} \pm 30.5$ ).

The diagnostic specimens were most often obtained from open biopsies, followed by aspiration and incision and drainage (Table 3). All 67 cases with fungal cultures showed positive results. Of the 53 histopathological analyses, the diagnostic specimens were obtained from open biopsies in 37 (69.8\%) cases, aspiration in $13(24.5 \%)$ cases, and incision and drainage in three $(5.7 \%)$ cases; positive results were obtained for 21 (of $37,56.8 \%$ ) cases, seven (of 13, 53.8\%) cases, and one (of three, $33.3 \%$ ) case, respectively. Positive histopathological analyses showed foreign-body giant cells and capsulated yeast-like organisms. The capsule structure was stained using periodic acid Schiff (PAS) stain in 22 (of 29, 75.9\%) patients, mucicarmine stain in 13 (of 29,44.8\%) patients, Gomori's Methenamine silver (GMS) stain in 18 (of 29, 62.1\%) patients, MassonFontana silver stain in case 15 , and colloidal iron techniques in case 23 .

Only two patients with immunocompetent status (cases 44 and 82) had their strains successfully identified, using cultures on dihydroxyphenylalanine (DOPA) and canavanine glycine bromothymol (CGB) blue agars; both patients were infected with CNVN. 
Table 2 | Epidemiological histories of 13 patients.

\begin{tabular}{|c|c|c|c|}
\hline Case no./References & Age/Sex & Epidemiological histories & Immune status \\
\hline 2/ Chleboun and Nade, 1977 & $40 / F$ & Contact with soil (farmer) & Sarcoidosis \\
\hline 52/ Kumlin et al., 1997 & $79 / \mathrm{M}$ & Contact with soil (farmer) & Lymphopenia \\
\hline 82/ Corral et al., 2011 & $65 / \mathrm{M}$ & $\begin{array}{l}\text { Contact with soil (agricultural worker), chronic trauma } \\
\text { (1 year) }\end{array}$ & Immunocompetent \\
\hline 38/ Dounis et al., 1991 & $55 / F$ & Chronic trauma & Immunocompetent \\
\hline 62/ Italiano et al., 2001 & $37 / F$ & Acute trauma & Sjogren's syndrome \\
\hline 68/ Wildstein et al., 2005 & $22 / \mathrm{M}$ & Chronic trauma (4 weeks) & Immunocompetent \\
\hline 26/ Zach and Penn, 1986 & $13 / F$ & Exposure to sea gull, chickens & Immunocompetent \\
\hline 39/ Abdul-Karim et al., 1991 & $9 / \mathrm{M}$ & Exposure to sparrows droppings & Interleukin-2 deficiency \\
\hline
\end{tabular}

\section{RADIOLOGICAL MANIFESTATIONS}

Of the 89 patients, $77(86.5 \%)$ had one or more radiological examinations of their affected bones. Sclerosis was observed in the relatively immunocompromised cases 23 and 45; periosteal reaction was described in 13 (of 76, 17.1\%) patients. Subperiosteal new bone formation was noted in case 79 (immunocompetent), and irregular cortical destruction and extensive periosteal reaction was noted in case 59 (relatively immunocompromised).

Of the 80 evaluable patients, osteomyelitis was found in 51 $(63.8 \%)$ patients. Case 41 was documented as having osteomyelitis, and the other 50 patients were diagnosed based on either a positive radiograph or bone scan; five (of 50, 10.0\%) patients were also diagnosed based on a positive histopathology. The presence of osteomyelitis among patients categorized as classically immunodeficient (11 of $18,61.1 \%$ ), relatively immunocompromised ( 20 of $30,66.7 \%$ ), and immunocompetent (20 of $32,62.5 \%$ ) did not differ $\left(\chi^{2}\right.$-test; $\left.p=0.911\right)$.

\section{DELAYS IN DIAGNOSIS}

Of the 88 evaluable patients (excluding case 36, that was diagnosed post-mortem), delays in diagnosis occurred among 20 (of $88,22.7 \%$ ) patients. The delayed time of these 20 patients (documented in only 14 patients) ranged from 6 days to 10 months. In addition, 13 (of 20,65.0\%) patients were initially misdiagnosed (Table 4), most commonly with tuberculosis (6 of 13, 46.2\%) primarily occurring in the vertebrae ( 5 of $6,83.4 \%$ ). The location of the source of discomfort was not reported for the remaining 7 (of 20,35.0\%) patients.

\section{TREATMENT, FOLLOW-UP ASSESSMENT, AND OUTCOMES}

Of the evaluable 87 patients (excluding patient 36 who did not receive any treatment and was diagnosed at autopsy as well as patient 59 whose treatment information was not available), 80 (of $87,92.0 \%$ ) patients received one treatment regimen and the other 7 (of 87, 8.0\%) patients changed treatments because their symptoms became aggravated or recurred.
Of the 80 patients who received only one treatment regimen, 3 (of $80,3.8 \%$ ) patients received surgery alone, 32 (of $80,40.0 \%$ ) patients received medical treatment alone and 45 (of 80, 56.2\%) patients received surgery in conjunction with medical treatment. Of the 3 patients that underwent surgery alone, 1 (of 3, 33.3\%) patient died and 2 (of $3,66.7 \%$ ) patients responded. Of the 32 patients treated with medical treatment alone, 10 patients (31.3\%) received monotherapy (AMB was the most commonly used treatment; 5 of 10, 50.0\%) and three patients (30.0\%) died. Twenty-two (of 32, 68.7\%) patients received combined therapies (AMB plus 5-FC was the most commonly used treatment; 14 of $22,36.4 \%)$, three of whom died (13.6\%). Of the 45 patients treated with surgery and medical treatments, 18 patients (40.0\%) underwent surgery and monotherapy (AMB was most commonly used; 10 of 18,55.6\%), one of whom died (5.6\%). A total of 27 (of $45,60.0 \%$ ) patients underwent surgery combined with several medicines (AMB plus 5-FU was the most commonly used treatment; 11 of $27,40.7 \%$ ), four of whom died (14.8\%).

In total, 68 patients responded to treatment, and 12 patients died (Table 5). Improvements of the symptoms and the rclinical signs of all 68 patients who responded were observed. Only six of these patients' (8.8\%) ESRs were measured after treatment, all of which were normal or decreased. Only 13 (of 68, 19.1\%) patients underwent serum cryptococcal antigen testing, and all of these patients showed reductions or undetectable levels. X-rays, CT, or MRI scans were obtained in 21 (of 68, 30.9\%) patients, and all of them showed healing or resolution. Bone scans were performed in two (of 68, 2.9\%) patients, and both of them presented reduced isotope uptake. Six of the 12 patients who received treatment but died were classically immunodeficient (50.0\%), five were relatively immunocompromised $(41.7 \%)$, and case 4 was immunocompetent (Table 6). In addition, nine (of 12, 75.0\%) patients presented with disseminated cryptococcosis. Only one of 12 patients' deaths was directly caused by cryptococcosis $(8.3 \%$; case 65 , relatively immunocompromised).

Of the seven patients who changed treatment, symptom aggravation during primary treatment occurred among five (of 


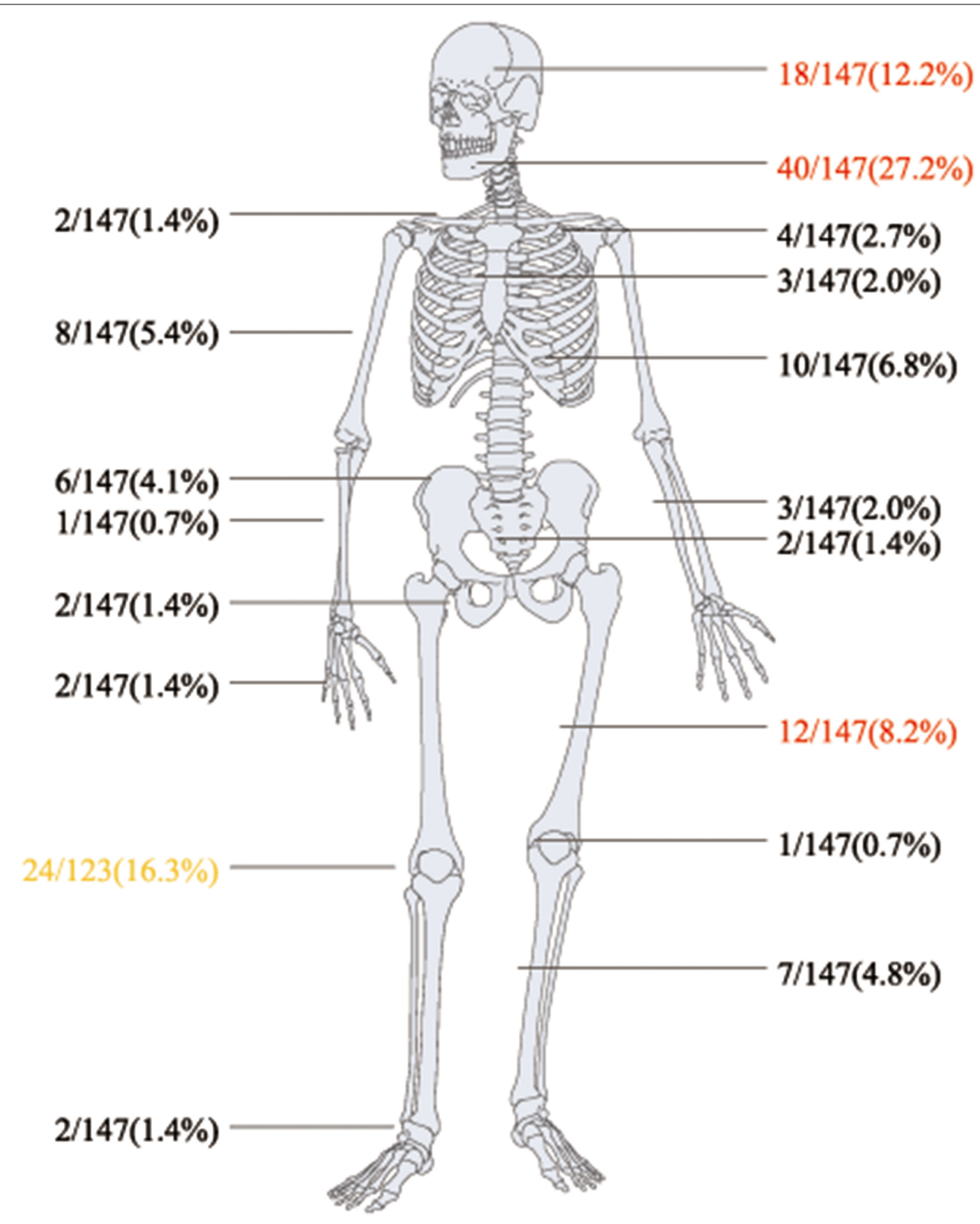

FIGURE 2 | Bones and joints involved (147 sites) in skeletal cryptococcal lesions.

seven, $71.4 \%$ ) patients, one of whom (case 22, relatively immunocompromised) underwent an ESR examination that revealed an ESR increase from $30 \mathrm{~mm} / \mathrm{h}$ to $>80 \mathrm{~mm} / \mathrm{h}$ after the administration of $917 \mathrm{mg}$ of AMB for 14 weeks. Symptoms recurred for case 7 (immunocompetent) and case 60 (relatively immunocompromised) after primary treatment, but neither met the criteria for relapse. Of these seven patients, one case (case 58) was categorized as classically immunodeficient, two were categorized as relatively immunocompromised, and the other four (57.1\%) were categorized as immunocompetent. Three (of seven, 42.9\%) patients presented with dissemination. All seven patients responded well to the subsequent treatment.
The follow-up time ranged between 2 weeks and 15 years (median $=13.5$ months); half of all patients (30 of 60) were followed-up for less than 1 year, and the other half were followedup for more than 1 year.

The factors associated with the overall skeletal cryptococcosis mortality rate, stratified by response to treatment, are listed in Table 7. Dissemination was a risk factor the overall mortality rate $(p=0.041)$; the patient immune status was not a risk factor mortality $(p=0.056)$.

Finally, to compare the mortality rates associated with different treatments, we analyzed 40 patients who were treated with AMB alone ( 2 of $5,40.0 \%)$, AMB plus 5 -FC (11 of $14,78.6 \%$ ), 


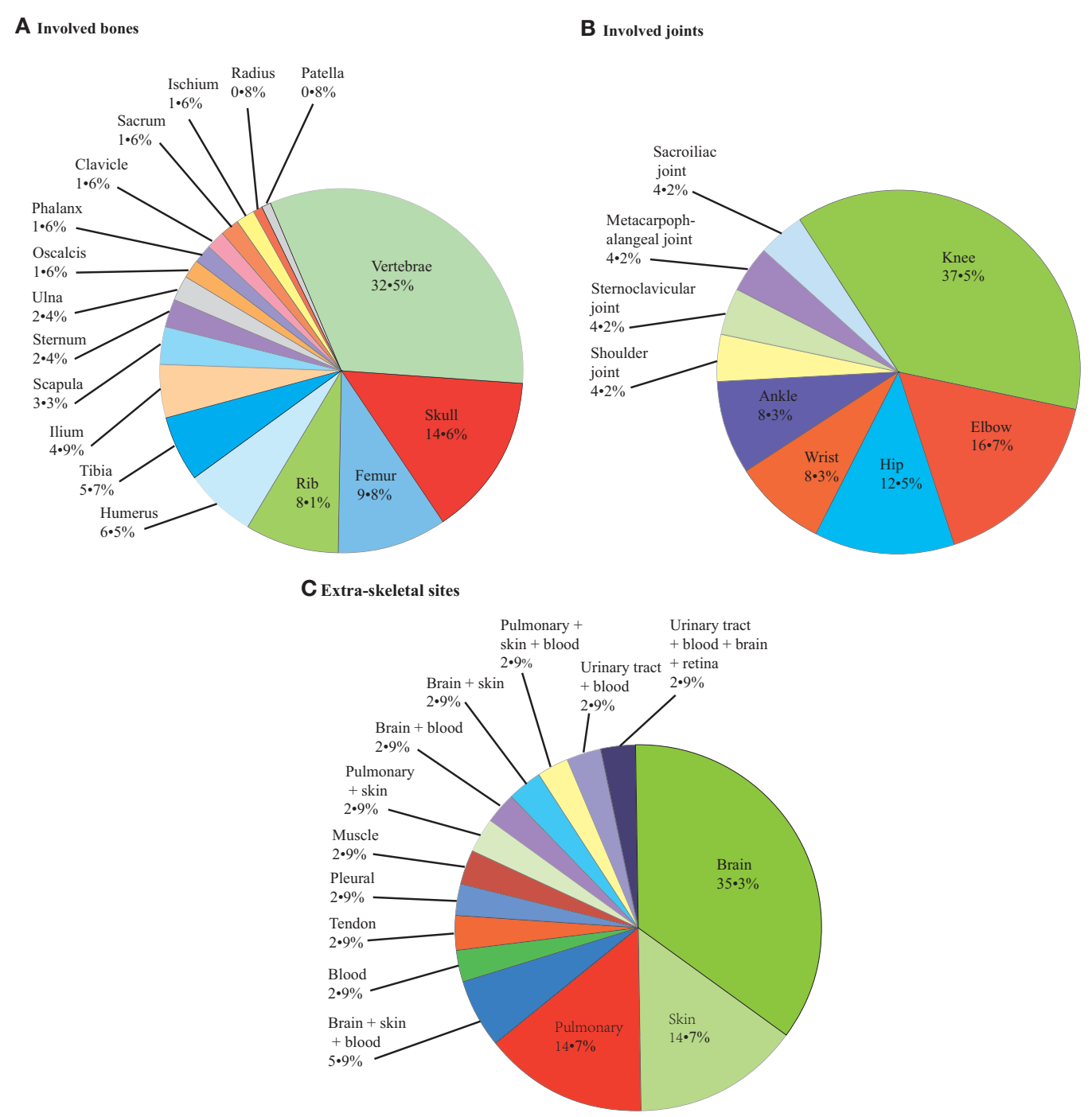

FIGURE 3 | Pie chart of patients' involved bones (A), joints (B) and extra-skeletal sites (C): 123, 24, 34, respectively.

Table 3 | Diagnostic modalities in 82 patients with skeletal cryptococcosis.

\begin{tabular}{llccr}
\hline Modality & $\begin{array}{l}\text { No. of } \\
\text { tests }\end{array}$ & Culture $\mathbf{n}(\%)$ & $\begin{array}{c}\text { Histopathological } \\
\text { analysis n (\%) }\end{array}$ & Both n (\%) \\
\hline Open biopsy & 50 & $13(26.0)$ & $10(20.0)$ & $27(54.0)$ \\
Aspiration & 27 & $14(51.9)$ & $5(18.5)$ & $8(29.6)$ \\
$\begin{array}{l}\text { Incision and } \\
\text { drainage }\end{array}$ & 5 & $2(40.0)$ & $0(0)$ & $3(60 / 0)$ \\
\hline Total No. & 82 & $29(35.4)$ & $15(18.3)$ & $38(46.3)$ \\
\hline
\end{tabular}

surgery combined with AMB (9 of $10,90.0 \%$ ), or surgery combined with $\mathrm{AMB}$ plus 5-FC (8 of $11,72.7 \%$ ); these cases were chosen because these three treatments (AMB, 5-FC and surgery) were utilized more often than other therapies. Specific information is listed in Table 8. The mortality rates of the four treatment regimens did not differ $(p=0.229)$, and dissemination predicted mortality $(p=0.044)$.

\section{DISCUSSION}

Because knowledge regarding the clinical features, treatment, and prognosis of skeletal cryptococcosis is limited, this large-scale systematic analysis of previously reported skeletal cryptococcosis was conducted to better understand the disease.

Skeletal cryptococcosis affects both individuals who are immunocompetent and those with abnormal immunity (Behrman et al., 1990; Zhang et al., 2012). Our study revealed immune status to be an important risk factor for this infection, which is consistent with previous reports (Hawkins and Armstrong, 1984; Jacobson et al., 2012). Most of the patients with immune abnormalities included in this study had defects of cellular immunity such as those related to lymphoma, leukemia, sarcoidosis, and long-term steroid use. Cellular immunity defects might predispose patients to cryptococcal infection, which can 


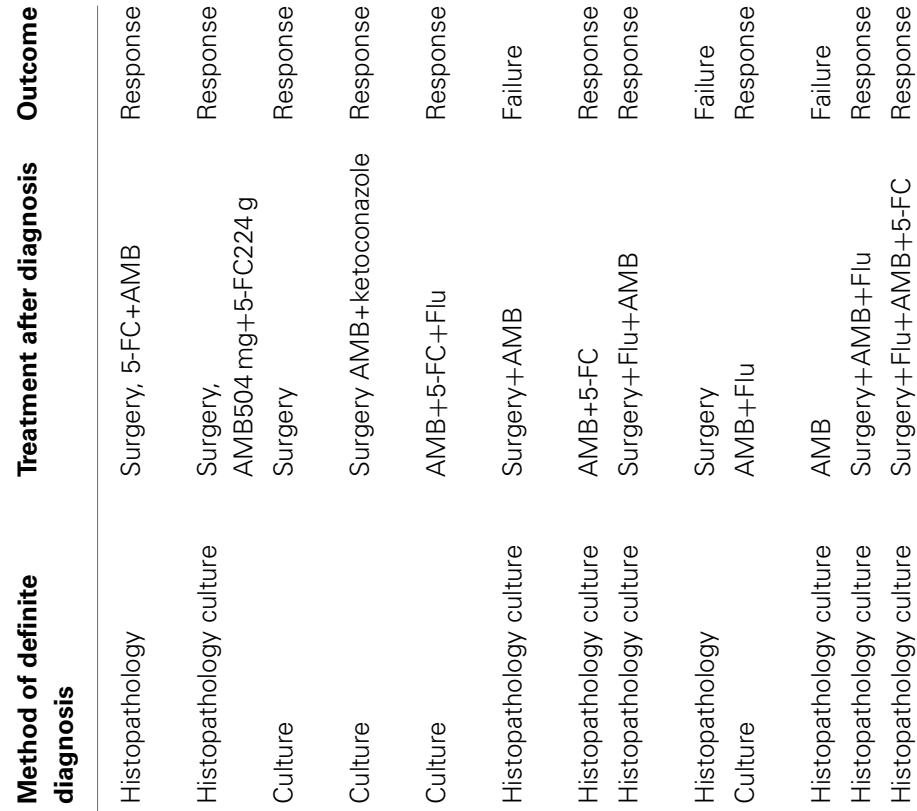

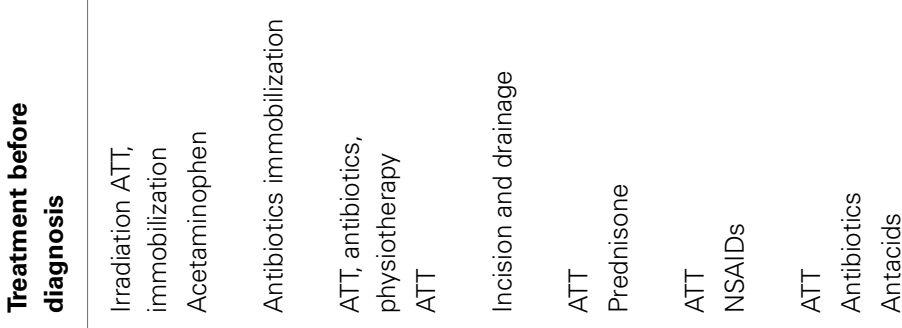

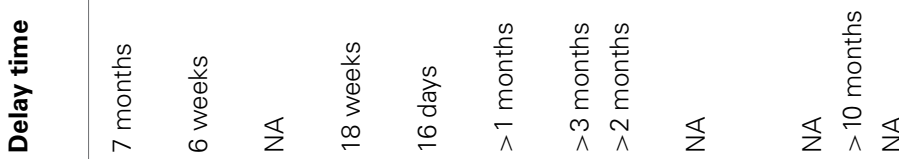

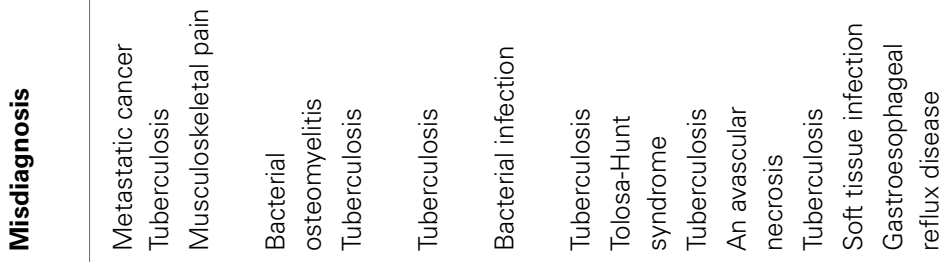

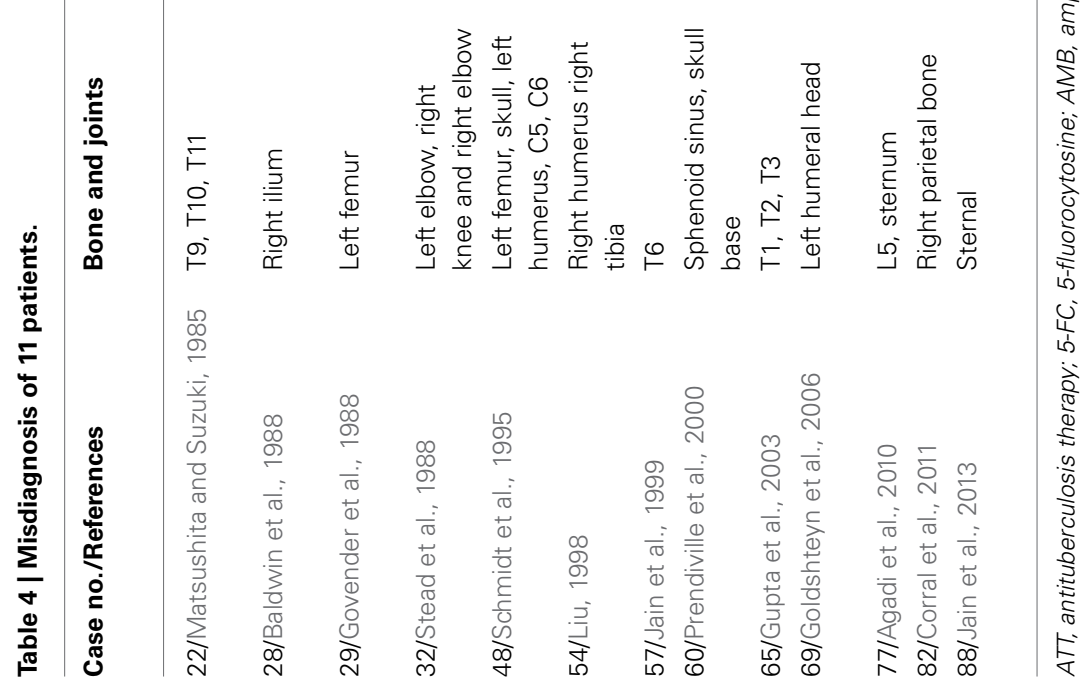


Table 5 | Treatment of the 80 patients.

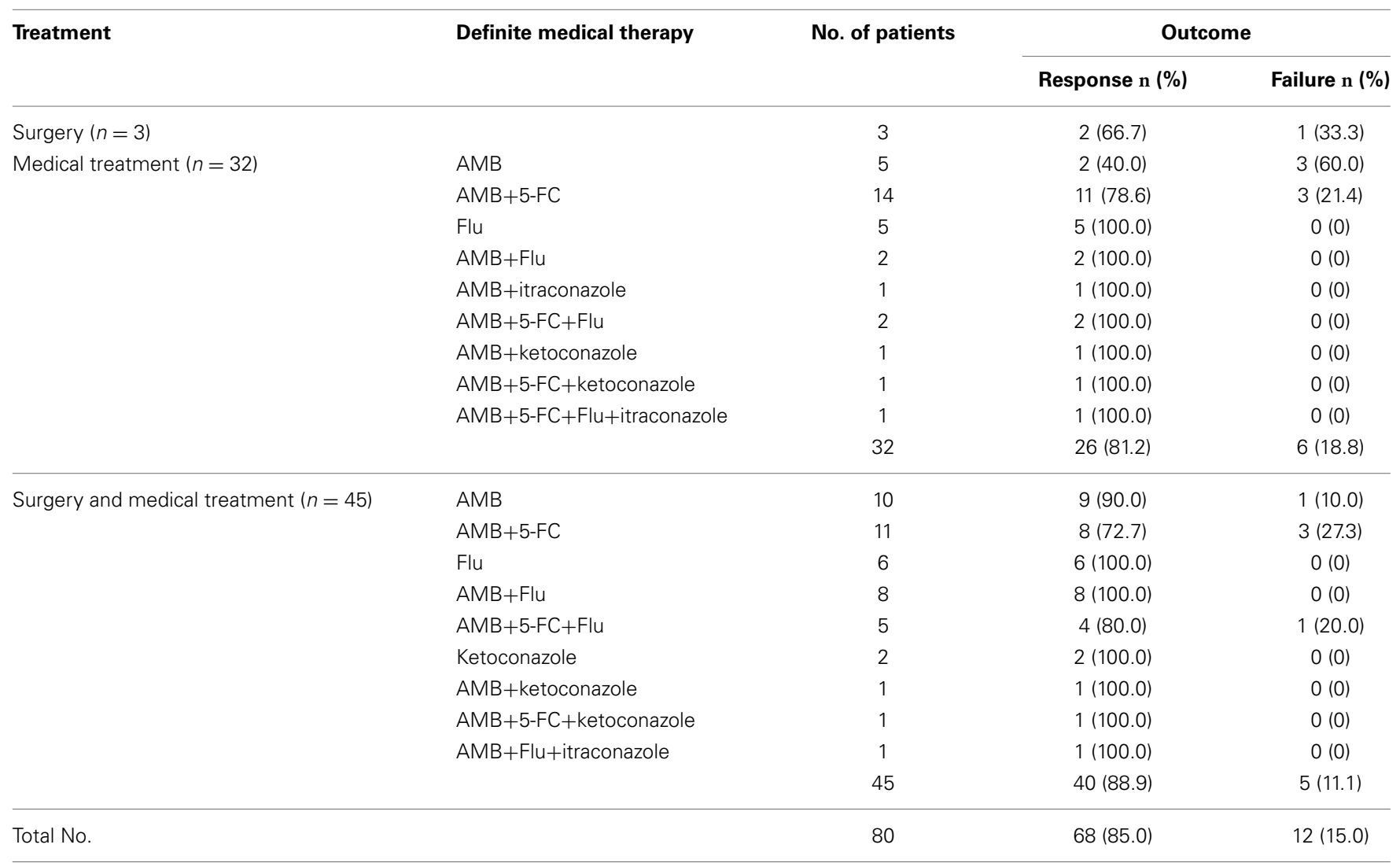

$A M B$, amphotericin B; 5-FC, 5-fluorocytosine; Flu, fluconazole.

lead to $\mathrm{T}$ cell abnormalities in hosts without other underlying diseases. This possibility suggests that $\mathrm{T}$ cell mediated immunity is the primary pathway for preventing cryptococcal infections (Meredith et al., 1979; Agadi et al., 2010; Jacobson et al., 2012). Thus, once a patient is suspected with cryptococcal infection, the evaluation of lymphocyte subsets, including counts and stimulation studies, should be routinely performed to specifically and sensitively reveal the patient immune status, as suggested by Wood and Miedzinski (1996).

Since the introduction of highly active antiretroviral therapy (HAART) in 1995, the mortality rate associated with AIDS has dramatically decreased (Mitchell and Perfect, 1995). Subsequently, steroids are considered the leading cause of skeletal cryptococcosis because of their extensive use for both therapeutic and recreational purposes (Benard et al., 1996; Hummel et al., 1996). In addition, the incidence of chronic diseases such as diabetes mellitus and hypertension has increased (Jain et al., 1999; Witte et al., 2000; Bruno et al., 2002). The number of patients with cryptococcal disease and who are classified as immunocompetent has risen greatly since 2004 and is estimated to increase by 0.2 per million every year (Zhang et al., 2012; Jain et al., 2013; Zhou et al., 2013). This increase was most likely due to the CNVG outbreak that originated in 1999 and resurged in 2004 (Chaturvedi and Chaturvedi, 2011). However, determining the reasons why patients with immunocompetent statuses were not found between 1999 and 2004 is difficult. CNVN, which is ecologically widespread and exists in soil contaminated by pigeon excreta, is more common in immunocompromised patients with cell-mediated immune deficiencies, whereas CNVG, which is traditionally found in eucalyptus trees located in tropical and subtropical areas (Speed and Dunt, 1995; Chaturvedi and Chaturvedi, 2011; Harris et al., 2011) (the south-eastern region of China is the most common location (Chen et al., 2008; Negroni, 2012), affects immunocompetent hosts. Thus, it is essential for clinical microbiology laboratories to accurately differentiate CNVG from other forms of $C$ neoformans to determine the final diagnosis and guide the initiation of or institute the appropriate treatment (Klein et al., 2009; Singh and Xess, 2010). Recently, a testing survey conducted by the New York State Department of Health indicated that only $5.0 \%$ of clinical laboratories were able to correctly identify CNVG (Klein et al., 2009). CNVN and CNVG are commonly differentiated by DOPA agar and CGB agar, where different colored reactions can be observed (Klein et al., 2009; Qadir et al., 2011). The results are often available within $48 \mathrm{~h}$; CNVG produces a blue color, whereas CNVN fails to cause a color change. Furthermore, Klein and colleagues first used the specific method of D2 large ribosomal subunit region sequencing to identify CNVG (Klein et al., 2009). McTaggart and colleagues explored a cost-effective method called matrix-assisted laser desorption/ionization time-of-flight mass spectrometry (MALDI-TOF MS) (McTaggart et al., 2011). Feng $\mathrm{X}$ provided a rapid, simple, and reliable method using Singleplex 
Table 6 | Twelve deceased patients.

\begin{tabular}{|c|c|c|c|c|c|}
\hline Case no./References & Age/Sex & $\begin{array}{l}\text { Disseminated } \\
\text { or not }\end{array}$ & Immune status & Treatment & Cause of death \\
\hline 1/ Chleboun and Nade, 1977 & $43 / \mathrm{M}$ & No & Relatively immunocompromised & Surgery & Unknown \\
\hline 4/ Chleboun and Nade, 1977 & $15 / \mathrm{M}$ & No & Immunocompetent & AMB395 mg & $\begin{array}{l}\text { Tuberculous } \\
\text { hepatitis and } \\
\text { staphylococcal } \\
\text { pneumonia }\end{array}$ \\
\hline 12/ Heenan and Dawkins, 1981 & $54 / \mathrm{M}$ & Yes & Classically immunodeficient & Surgery AMB+5-FC2700 g & Unknown \\
\hline 16/ Cash and Goodman, 1983 & $59 / \mathrm{M}$ & CNS & Relatively immunocompromised & $\mathrm{AMB}+5-\mathrm{FC}$ & $\begin{array}{l}\text { Cardiorespiratory } \\
\text { arrest on the } \\
\text { 13th day of } \\
\text { therapy }\end{array}$ \\
\hline 17/ Perfect et al., 1983 & $46 / F$ & $\begin{array}{l}\text { CNS, skin, } \\
\text { blood }\end{array}$ & Classically immunodeficient & $\mathrm{AMB}+5-\mathrm{FC}$ & $\begin{array}{l}\text { Serratia } \\
\text { septicemia }\end{array}$ \\
\hline 18/ Perfect et al., 1983 & $32 / F$ & $\begin{array}{l}\text { Retina, CNS, } \\
\text { urine, blood }\end{array}$ & Classically immunodeficient & AMB & Unknown \\
\hline 21/ Reinig et al., 1984 & $10 / F$ & No & Classically immunodeficient & Surgery $A M B+5-F C$ & $\begin{array}{l}\text { Respiratory } \\
\text { failure }\end{array}$ \\
\hline 27/ Ricciardi et al., 1986 & $37 / \mathrm{M}$ & $\begin{array}{l}\text { CNS, skin, } \\
\text { blood }\end{array}$ & Classically immunodeficient & $A M B+5-F C$ & Unknown \\
\hline 54/ Liu, 1998 & $60 / \mathrm{M}$ & Yes & Relatively immunocompromised & Surgery AMB & $\begin{array}{l}\text { Severe hepatic } \\
\text { failure }\end{array}$ \\
\hline 65/ Gupta et al., 2003 & $24 / F$ & Yes & Relatively immunocompromised & Surgery AMB+5-FC & Cryptococcosis \\
\hline 70/ Goldshteyn et al., 2006 & $84 / F$ & CNS & Classically immunodeficient & Surgery $\times 2 \mathrm{AMB}+5-\mathrm{FC}+\mathrm{Flu}$ & Unknown \\
\hline 77/ Agadi et al., 2010 & $29 / F$ & Yes & Relatively immunocompromised & AMB & Cardiac failure \\
\hline
\end{tabular}

AMB, amphotericin B; 5-FC, 5-fluorocytosine; Flu, fluconazole.

Table 7 | Factors associated with overall skeletal cryptococcosis mortality (excluding cases 25, 36, and 59 and the 12 deceased patients).

\begin{tabular}{lccc}
\hline Characteristics & Non-survival (\%) & Survival (\%) & $P$-value \\
\hline N & 67 & 12 & \\
Mean age & $39.2 \pm 19.1$ & $41.1 \pm 21.1$ & 0.752 \\
No. Male & $33(49.3 \%)$ & $5(41.7 \%)$ & 0.628 \\
Epidemiological histories & $11(16.4 \%)$ & $0(0)$ & 0.289 \\
Multiple site infections & $23(34.3 \%)$ & $6(50.0 \%)$ & 0.476 \\
Extra-skeletal infections & $25(37.3 \%)$ & $6(50.0 \%)$ & 0.612 \\
Dissemination & $28(41.8 \%)$ & $9(75.0 \%)$ & 0.034 \\
Immune abnormality & $40(59.7 \%)$ & $11(91.7 \%)$ & 0.071 \\
Delay in diagnosis & $14(20.9 \%)$ & $3(25.0 \%)$ & 1.000 \\
\hline
\end{tabular}

PCR assay that is suitable for laboratory diagnoses and largescale epidemiologic studies (Feng et al., 2013). Both strains were shown to have similar susceptibilities to antifungal drugs when tested in vitro in some reports (Chen et al., 2000; Thompson et al., 2009). The treatments for patients with disseminated disease due to CNVG are the same as those for CNVN.

Our study did not reveal a relationship between diagnosis and sex, which is consistent with previous reports (Behrman et al., 1990; Wood and Miedzinski, 1996; Zhou et al., 2013). However, Behrman and colleagues reported that $51.0 \%$ (20 of 39) patients were males in 1990 (Behrman et al., 1990), whereas Bruno and colleagues reported that $61.9 \%$ (13 of 21 ) patients were males
Table 8 | Factors associated with 40 patients treated with AMB alone, $A M B$ plus 5-FU, surgery combined with $A M B$, or surgery combined with AMB plus 5-FU.

\begin{tabular}{lccc}
\hline Characteristics & Non-survival (\%) & Survival (\%) & $\boldsymbol{P}$-value \\
\hline $\mathrm{N}$ & 30 & 10 & \\
Mean age & $36.2 \pm 22.4$ & $36.6 \pm 17.8$ & 0.964 \\
No. Male & $16(53.3 \%)$ & $4(40.0 \%)$ & 0.465 \\
Epidemiological histories & $5(16.7 \%)$ & $0(0)$ & 0.408 \\
Multiple site infections & $8(26.7 \%)$ & $6(60.0 \%)$ & 0.126 \\
Extra-skeletal infections & $11(36.7 \%)$ & $5(50.0 \%)$ & 0.709 \\
Dissemination & $11(36.7 \%)$ & $8(80.0 \%)$ & 0.044 \\
Immune abnormality & $16(53.3 \%)$ & $9(90.0 \%)$ & 0.090 \\
Delay in diagnosis & $7(23.3 \%)$ & $3(30.0 \%)$ & 1.000 \\
Treatment AMB & $2(6.7 \%)$ & $3(30.0 \%)$ & 0.229 \\
\multicolumn{1}{c}{ AMB+5-FC } & $11(36.7 \%)$ & $3(30.0 \%)$ & \\
surgery+AMB & $9(30.0 \%)$ & $1(10.0 \%)$ & \\
\multicolumn{1}{c}{ surgery+AMB+5-FC } & $8(26.7 \%)$ & $3(30.0 \%)$ & \\
\end{tabular}

in 2002 (Bruno et al., 2002); these findings might be due to the smaller number of reviewed cases in their studies. Cryptococcal infections occurred in all age groups, with a slight tendency toward for younger people to be affected (e.g., most patients were under 45 years of age). However, the patients with relatively immunocompromised statuses were elderly, which might be explained by the aforementioned leading cause of skeletal 
cryptococcosis $(\mathrm{CNVN})$ and the fact that older patients are more likely to have chronic diseases.

Skeletal cryptococcosis is usually secondary to hematogenous migration from a primary pulmonary infection after inhaling microscopic, airborne fungal spores (especially after exposure to soil or poultry). These spores are a cause for cryptococcal infection (Armonda et al., 1993; Wood and Miedzinski, 1996). Direct inoculation during trauma is also possible (Chleboun and Nade, 1977; Dounis et al., 1991; Italiano et al., 2001). However, only $14.8 \%$ of the patients included in this review had contact with soil or pigeons or a history of trauma; incomplete patient histories might explain this finding. In addition, host immunity did not significantly affect their epidemiological histories. This result differs from that of Jacobson and colleagues, who reported that host immunity effectively excluded infection after initial exposure (Jacobson et al., 2012). This disparity might be due to the recent increase in patients with immunocompetent statuses. Other infectious pathways include direct inoculation through the skin during trauma and contiguous spread combined with the lower involvement of the lymphatic route (Zanelli et al., 2001).

Compared with most of the previous articles that reviewed skull cryptococcosis, articular cryptococcosis, or other bony cryptococcosis alone, such as in Chleboun and Nade's (1977) study, we examined all types of involved sites (Chleboun and Nade, 1977). Any bone or joint can be affected, but the most common site was the vertebrae, which is consistent with Chleboun's report (Chleboun and Nade, 1977); the sufficient blood supply of the vertebrae might explain this finding. The second and third most common sites have changed from the pelvis and rib in 1977 to the skull and femur (Chleboun and Nade, 1977). The most common affected joint was the knee, which is consistent with the study by Bruno et al. (2002). The involvement of multiple bones occurred more regularly in adjacent areas than discrete areas, which indicates the extension of local foci; this result is consistent with Behrman and colleagues' study in 1990 (Behrman et al., 1990). Patients with classically immunodeficient statuses were most likely to have concurrent extra-skeletal involvement sites, and meningitis was the most common extra-skeletal infection. Overall, $47.7 \%$ of patients with (especially the classically immunodeficient status) or without immune abnormalities presented with dissemination, and these patients were more likely to show symptom aggravation, recurrence, or death.

The characteristic symptoms of skeletal cryptococcosis are pain and swelling (Chleboun and Nade, 1977; Behrman et al., 1990; Wood and Miedzinski, 1996). Fever, which is not a primary patient complaint (Behrman et al., 1990), was found in only $20.9 \%$ of evaluable patients comparable with a previous study, which reported a rate of $18.0 \%$ (Wood and Miedzinski, 1996). In addition, classic symptoms such as vomiting (Cash and Goodman, 1983; Agadi et al., 2010), blurred vision (Cash and Goodman, 1983; Prendiville et al., 2000; Ching et al., 2004), dizziness, seizure, diplopia, trismus (Cash and Goodman, 1983), limited motion (Bunning and Barth, 1984; Ricciardi et al., 1986; Sinnott and Holt, 1989), paralysis (Meredith et al., 1979; Gupta et al., 2003), muscle weakness, urinary retention (Gurevitz et al., 1994), and sciatica (Houda et al., 2011)can occur among patients with cryptococcal infections of specific sites; the location can assist in making the final diagnosis.

The ESR can be elevated to various levels when the infection is found in the bone, decline to normal when osseous lesions heal, and increase again when patients have an extensive relapse (Chleboun and Nade, 1977; Behrman et al., 1990; Wood and Miedzinski, 1996).

The diagnosis of skeletal cryptococcosis is primarily based on the examination of lesion specimens from aspiration, surgery, and open biopsies (Behrman et al., 1990; Wood and Miedzinski, 1996; Gupta et al., 2003). Aspiration was the most common method performed in our review, whereas Behrman and colleagues reported that open biopsy was the most commonly performed technique (Behrman et al., 1990); this discrepancy might be attributable to advancements in medical techniques since 1990. All of these methods have a similar diagnostic value. Thus, aspiration is recommended first given its increased convenience and minimal harm caused to the body; however, if aspiration specimens fail to yield diagnostic value, then open biopsy is recommended. All samples should be sent for culture, smear, and histology examinations (Wood and Miedzinski, 1996). Culture is the gold standard diagnosis (Wood and Miedzinski, 1996). After staining with India ink, the organism resembles cells with a halo due to a lack of capsule staining, and it is easily detected using specific PAS, mucicarmine, and GMS stains (although it is poorly stained by $\mathrm{H} \& \mathrm{E}$ ). Urease-positive mucoid colonies are produced in cultures on SDA agar usually within 3-5 days (Mitchell and Perfect, 1995; Qadir et al., 2011; Jain et al., 2013). Currently available commercial methods for yeast identification, such as API 20 AUX (bioMerieux, Paris, France) and Vitek (bioMerieux), are used to identify the yeast-like organisms (Qadir et al., 2011; Zhou et al., 2013). Once the organisms are detected, identifying the strain is recommended as mentioned above.

Examinations for disseminated cryptococcosis should be performed after identification. Relevant examinations generally consist of the following procedures: lumbar puncture for antigen testing and culture, blood culture, urine culture, sputum culture, and skin lesion culture (Wood and Miedzinski, 1996). Recently, a marrow aspirate was considered in the diagnosis of disseminated cryptococcosis (Venkatachala et al., 2010). Testing for serum cryptococcal antigen using a latex agglutination test (LA), an enzyme immunoassay (EIA) or lateral flow assay (LFA) is useful for diagnosis given their sensitivity and specificity (Bruno et al., 2002; Lindsley et al., 2011; Hansen et al., 2013). However, serum cryptococcal antigen is not always positive even when infection is demonstrated via culture (Hawkins and Flaherty, 2007). CSF cryptococcal antigen testing is more highly sensitive and specific for meningitis than serum cryptococcal antigen testing (Hawkins and Flaherty, 2007).

The radiological findings of skeletal cryptococcus were nonspecific (Chleboun and Nade, 1977; Behrman et al., 1990; Wood and Miedzinski, 1996); sclerosis or periosteal reaction, which are typical symptoms associated with tumors, were found in our study (Levine et al., 1985; Bosch et al., 1994; Witte et al., 2000; McGuire et al., 2011). Furthermore, patients with poor immune status were less likely to show radiological features of malignancy. The differential diagnoses based on radiological features included 
microbial infections, namely Staphylococcus aureus, Brucella, Actinomyces, tuberculosis, and neoplasms such as Ewing's sarcoma, osteogenic sarcoma, enchondroma, and giant cell tumor (Behrman et al., 1990; Witte et al., 2000). In addition, the diagnosis of osteomyelitis is commonly indicated based on radiological studies that are non-specific for $C$ neoformans (Behrman et al., 1990), and our results showed that patients can contract osteomyelitis regardless of the presence of immune abnormalities. Radiological studies should be routinely performed because they assist the final diagnosis and can be used as a monitoring index to detect the efficacy of therapy based on radiological improvement, healing, or resolution.

The insidious course of this disease contributes to the delays in diagnosis (Bunning and Barth, 1984; Matsushita and Suzuki, 1985; Baldwin et al., 1988). Importantly, tuberculosis was the most common reason for misdiagnosis. Although our results indicated that delayed diagnosis did not contribute to a worse survival rate, clinicians must be alerted to this disease and identify it in a timely manner.

Except for the lungs ans CNS, no standardized treatment protocol exists for cryptococcal infection for specific body sites (Jain et al., 2013; Ramkillawan et al., 2013). For these sites, surgery in conjunction with antifungals, antifungals alone, or (rarely) surgery alone have been demonstrated to be effective. According to the Infectious Disease Society of America (IDSA), surgery, which effectively and rapidly eliminates the fungal burden and prevents the contiguous spread of infection (Chleboun and Nade, 1977; Govender et al., 1988; McGuire et al., 2011), should be performed to patients with persistent or refractory bone disease (Perfect et al., 2010). Surgery also provides physicians with the opportunity to obtain specimens for histological and microbiological examination to make a definitive diagnosis (Ramkillawan et al., 2013). The selection of antifungal agents and the duration of therapy depends on factors including disease severity, host immune status, the infection site, and therapeutic response (Qadir et al., 2011; Zhang et al., 2012). Systemic therapy consists of AMB, 5-FC, fluconazole, ketoconazole, or some combination therein (Bryan, 1977; Galloway and Schochet, 1981; Stead et al., 1988; Ueda et al., 1992; Perfect et al., 2010). Although, combination therapy with $\mathrm{AMB}$ and 5-FC (with or without surgery) did not outperform AMB alone (with or without surgery) in terms of improving the mortality rate (which might be due to the small number of cases), combined therapy is recommended. This result is contrary to previous reports (Bryan, 1977; Poliner et al., 1979; Shaff et al., 1982; Raftopoulos et al., 1998; Perfect et al., 2010) and might be due to the small number of cases reviewed here. Thus, combined therapy is recommended given the prevention of secondary drug resistance, the shorter duration of therapy, smaller total dosage, and the reduced likelihood of side effects (Bryan, 1977; Raftopoulos et al., 1998; Jain et al., 2011). The most common treatment is a combination of AMB and 5-FC, which can decrease the high nephro- and hepatotoxicity of AMB (Bruno et al., 2002). The lipid formulation of AMB is used in patients with renal impairments (Perfect et al., 2010). The IDSA indicates that 200-400 mg per day of oral fluconazole for 6-12 months is the treatment of choice for patients with immunocompetent status and non-meningeal, non-pulmonary cryptococcosis because of its significantly reduced toxicity (Agadi et al., 2010; Perfect et al., 2010; Qadir et al., 2011; Zhou et al., 2013). Several case reports published over the last decade have demonstrated the successful treatment of cryptococcal osteomyelitis using fluconazole alone (Hummel et al., 1996; Wildstein et al., 2005; Agadi et al., 2010; Qadir et al., 2011; Zhou et al., 2013). Patients with disseminated cryptococcosis had unfavorable outcomes in our study, and this result is consistent with previous reports (Behrman et al., 1990; Bruno et al., 2002; Hawkins and Flaherty, 2007). Combination induction therapy of $\mathrm{AMB}$ and 5-FC followed by consolidation and maintenance therapies with fluconazole are recommended for patients with disseminated cryptococcosis (Perfect et al., 2010; Zhang et al., 2012). Suppressive treatments for disseminated disease due to CNVG are the same as those for CNVN described above. The ultimate duration of therapy is unknown, but it should be based on clinical findings, ESRs, serum cryptococcal antigen levels, and radiological improvements (Goldshteyn et al., 2006; Zhang et al., 2012).

The outcomes of patients with disseminated cryptococcosis were unfavorable, and those of patients with or without immune abnormalities were similar; these findings differ from previous studies (Corral et al., 2011; Jou et al., 2011; Qadir et al., 2011; Jain et al., 2013). This disparity might be explained by the recent CNVG outbreak. The recurrence rate of skeletal cryptococcosis is low (Hawkins and Flaherty, 2007). However, unlike the successful treatment of cryptococcal meningoencephalitis (demonstrated via CSF culture) and that of pulmonary cryptococcosis (demonstrated via sputum culture or the specimens obtained during bronchoscopy) (Perfect et al., 2010), it is difficult to prove the success of primary therapy in skeletal cryptococcus. Hence, once cannot distinguish relapse from recurrence. Clinical and radiographical follow-up assessments, as well as serum cryptococcal antigens, should be monitored carefully. For the qualitative or quantitative detection of serum cryptococcal antigen, a latex agglutination test (LA), an enzyme immunoassay (EIA) or a LFA should be used, and LFA shows excellent overall agreement with EIA (Lindsley et al., 2011; Hansen et al., 2013).Once an abnormal manifestation occurs during the primary therapy, a larger total dosage is recommended. If the abnormal manifestation recurs, then susceptibility testing should be performed to formulate the best therapy by evaluating the changes in the minimum inhibitory concentration (MIC) of the recurrent isolates and original isolates (Perfect et al., 2010). Prednisone prescribed for other diseases should be tapered during skeletal cryptococcosis treatment, given the drug's effect on immunity (Noh et al., 1999).

\section{CONCLUDING REMARKS}

Skeletal cryptococcosis occurs in patients with immune abnormalities and even in those who are immunocompetent. An immune abnormality is a risk factor but it does not predict mortality. Likewise, neither immunocompetence nor immune abnormalities predicted the deaths caused by recent CNVG outbreaks. Patients with (especially classic immunodeficiencies) or without immune abnormalities present with dissemination, and these patients are more likely to have unfavorable prognoses. Clinicians must be alert to this disease and be able to identify the particular fungal strain. No standardized treatment protocol 
exists for skeletal cryptococcosis. Although, combination therapy with AMB and 5-FC (with or without surgery) did not outperform AMB alone (with or without surgery) in terms of improving the mortality rate (which might be due to the small number of cases reported), combined therapy is recommended. Given that our series was unable to collect all information (which led to difficulties in further elucidating this disease), creating a disease database of skeletal cryptococcosis is recommended.

\section{AUTHOR CONTRIBUTIONS}

Heng-Xing Zhou and $\mathrm{Lu} \mathrm{Lu}$, as first coauthors, contributed equally to drafting and revising the review with input from all authors. All authors approved the final version.

\section{ACKNOWLEDGMENTS}

The authors are grateful to Dr. Xiaohong Kong for critically reading the manuscript and helpful discussions.

\section{REFERENCES}

Abdul-Karim, F. W., Pathria, M. N., Heller, J. G., and Sorensen, R. U. (1991). Case report 664. Cryptococcus neoformans osteomyelitis. Skeletal Radiol. 20, 227-229. doi: 10.1007/BF00241676

Agadi, J. B., Madni, N. A., Nanjappa, V., and Govindaiah, H. K. (2010). Cryptococcal osteomyelitis of the skull in a patient with transient lymphopenia. Neurol. India 58, 300-302. doi: 10.4103/0028-3886.63798

Al-Tawfiq, J. A., and Ghandour, J. (2007). Cryptococcus neoformans abscess and osteomyelitis in an immunocompetent patient with tuberculous lymphadenitis. Infection 35, 377-382. doi: 10.1007/s15010-007-6109-9

Amenta, P. S., Stead, J., and Kricun, M. E. (1983). Case report 226: isolated Cryptococcus neoformans osteomyelitis of femur. Skeletal Radiol. 9, 263-265. doi: 10.1007/BF00354129

Amit, A., Sudish, K., and Pople, I. K. (2008). Primary calvarial cryptococcal osteomyelitis in a patient with idiopathic lymphopenia. Acta Neurochir. (Wien) 150, 713-714. doi: 10.1007/s00701-008-1608-8

Armonda, R. A., Fleckenstein, J. M., Brandvold, B., and Ondra, S. L. (1993). Cryptococcal skull infection: a case report with review of the literature. Neurosurgery 32, 1034-1036. doi: 10.1227/00006123-199306000-00028

Baldwin, S., Stagno, S., Odrezin, G. T., Kelly, D. R., and Whitley, R. J. (1988). Isolated Cryptococcus neoformans osteomyelitis in an immunocompetent child. Pediatr. Infect. Dis. J. 7, 289-292. doi: 10.1097/00006454-198804000-00011

Behrman, R. E., Masci, J. R., and Nicholas, P. (1990). Cryptococcal skeletal infections: case report and review. Rev. Infect. Dis. 12, 181-190. doi: 10.1093/clinids/12.2.181

Benard, G., Gryschek, R. C., Duarte, A. J., and Shikanai-Yasuda, M. A. (1996). Cryptococcosis as an opportunistic infection in immunodeficiency secondary to paracoccidioidomycosis. Mycopathologia 133, 65-69. doi: 10.1007/BF00439115

Bosch, X., Ramón, R., Font, J., Alemany, X., and Coca, A. (1994). Bilateral cryptococcosis of the hip. A case report. J. Bone. Joint. Surg. Am. 76, 1234-1238.

Brand, C., Warren, R., Luxton, M., and Barraclough, D. (1985). Cryptococcal sacroiliitis. Ann. Rheum. Dis. 44, 126-127. doi: 10.1136/ard.44.2.126

Bruno, K. M., Farhoomand, L., Libman, B. S., Pappas, C. N., and Landry, F. J. (2002). Cryptococcal arthritis, tendinitis, tenosynovitis, and carpal tunnel syndrome: report of a case and review of the literature. Arthritis Rheum. 47, 104-108. doi: 10.1002/art1.10249

Bryan, C. S. (1977). Vertebral osteomyelitis due to Cryptococcus neoformans. Case report. J. Bone Joint Surg. Am. 59, 275-276.

Bunning, R. D., and Barth, W. F. (1984). Cryptococcal arthritis and cellulitis. Ann. Rheum. Dis. 43, 508-510. doi: 10.1136/ard.43.3.508

Burton, R., Gogela, N., Rebe, K., McNally, M., and Meintjes, G. (2009). Cryptococcal immune reconstitution inflammatory syndrome presenting with erosive bone lesions, arthritis and subcutaneous abscesses. AIDS 23, 2371-2373. doi: 10.1097/QAD.0b013e328330975f

Case Records of the Massachusetts General Hospital. (1999). Weekly Clinicopathological Exercises. Case 19-1999, A 55-year-old man with a destructive bone lesion 17 months after liver transplantation. New. Engl. J. Med. 340, 1981-1988. doi: 10.1056/NEJM199906243402508
Cash, J. B., and Goodman, N. L. (1983). Cryptococcal infection of the temporal bone. Diagn. Microbiol. Infect. Dis. 1, 257-260. doi: 10.1016/07328893(83)90025-1

Chang, W. C., Tzao, C., Hsu, H. H., Chang, H., Lo, C. P., and Chen, C. Y. (2005). Isolated cryptococcal thoracic empyema with osteomyelitis of the rib in an immunocompetent host. J. Infect. 51, e117-e119. doi: 10.1016/j.jinf.2004.10.011 Chaturvedi, V., and Chaturvedi, S. (2011). Cryptococcus gattii: a resurgent fungal pathogen. Trends. Microbiol. 19, 564-571. doi: 10.1016/j.tim.2011.07.010

Chen, J., Varma, A., Diaz, M. R., Litvintseva, A. P., Wollenberg, K. K., and KwonChung, K. J. (2008). Cryptococcus neoformans strains and infection in apparently immunocompetent patients, China. Emerging Infect. Dis. 14, 755-762. doi: 10.3201/eid1405.071312

Chen, Y. C., Chang, S. C., Shih, C. C., Hung, C. C., Luhbd, K. T., Pan, Y. S., et al. (2000). Clinical features and in vitro susceptibilities of two varieties of Cryptococcus neoformans in Taiwan. Diagn. Microbiol. Infect. Dis. 36, 175-83. doi: 10.1016/S0732-8893(99)00137-6

Ching, N., Lasky, J., Lazareff, J., Moore, T. B., and Nielsen, K. (2004). Enlarging parietal mass with lytic skull lesion. Pediatr. Infect. Dis. J. 23, 589, 595-589, 596.

Chleboun, J., and Nade, S. (1977). Skeletal cryptococcosis. J. Bone Joint Surg. Am. 59, 509-514.

Cook, P. P. (2001). Successful treatment of cryptococcal osteomyelitis and paraspinous abscess with fluconazole and flucytosine. South Med. J. 94, 936-938. doi: 10.1097/00007611-200109000-00024

Corral, J. E., Lima, S., Quezada, J., Samayoa, B., and Arathoon, E. (2011). Cryptococcal osteomyelitis of the skull. Med. Mycol. 49, 667-671. doi: 10.3109/ 13693786.2011.558124

Dounis, E., Giamarellou, H., Peppas, T., and Sfikakis, P. (1991). Skull, patella and thigh cryptococcosis after a crashing injury of the temporal bone. J. Chemother. 3, 130-133.

Feng, X., Fu, X., Ling, B., Wang, L., Liao, W., and Yao, Z. (2013). Development of a singleplex PCR assay for rapid identification and differentiation of Cryptococcus neoformans var. grubii, Cryptococcus neoformans var. neoformans Cryptococcus gattii, and hybrids. J. Clin. Microbiol. 51, 1920-1923. doi: 10.1128/JCM.0006413

Fialk, M. A., Marcove, R. C., and Armstrong, D. (1981). Cryptococcal bone disease: a manifestation of disseminated cryptococcosis. Clin. Orthop. Relat. Res. 158, 219-223.

Flannery, M. T., Rivera, L., and Casanas, B. (2012). Cryptococcal neoformans osteomyelitis-a case report and review of literature. Med. Case Rep. 3, 23-25.

Galloway, D. C., and Schochet, S. S. (1981). Cryptococcal skull granuloma. Case report. J. Neurosurg. 54, 690-692. doi: 10.3171/jns.1981.54.5.0690

Geller, D. S., Pope, J. B., Thornhill, B. A., and Dorfman, H. D. (2009). Cryptococcal pyarthrosis and sarcoidosis. Skeletal. Radiol. 38, 721-727. doi: 10.1007/s00256009-0691-0

Glynn, M. J., Duckworth, G., Ridge, J. A., Grange, W. J., and Gibbs, D. D. (1994). Cryptococcal spondylitis: solitary infective bone lesions are not always tuberculous. Rheumatology 33, 1085-1086. doi: 10.1093/rheumatology/33.11.1085

Goldshteyn, N., Zanchi, A., Cooke, K., and Agha, R. (2006). Cryptococcal osteomyelitis of the humeral head initially diagnosed as avascular necrosis. South. Med. J. 99, 1140-1141. doi: 10.1097/01.smj.0000224744.75040.13

Gould, P. R., and Gould, I. M. (1985). Cryptococcosis in Zimbabwe. Trans. R. Soc. Trop. Med. Hyg. 79, 67-69. doi: 10.1016/0035-9203(85)90239-1

Govender, S., Ganpath, V., Charles, R. W., and Cooper, K. (1988). Localized osseous cryptococcal infection. Report of 2 cases. Acta. Orthop. Scand. 59, 720-722. doi: 10.3109/17453678809149434

Gupta, S. K., Chhabra, R., Sharma, B. S., Das, A., and Khosla, V. K. (2003). Vertebral cryptococcosis simulating tuberculosis. Br. J. Neurosurg. 17, 556-571. doi: 10.1080/02688690310001626868

Gurevitz, O., Goldschmied-Reuven, A., Block, C., Kopolovic, J., Farfel, Z., and Hassin, D. (1994). Cryptococcus neoformans vertebral osteomyelitis. J. Med. Vet. Mycol. 32, 315-318. doi: 10.1080/02681219480000391

Hammerschlag, M. R., Domingo, J., Haller, J. O., and Papayanopulos, D. (1982). Cryptococcal osteomyelitis: report of a case and a review of the literature. Clin. Pediatr. 21, 109-112. doi: 10.1177/000992288202100208

Hansen, J., Slechta, E. S., Gates-Hollingsworth, M. A., Neary, B., Barker, A. P., Bauman, S., et al. (2013). Large-scale evaluation of the immuno-mycologics lateral flow and enzyme-linked immunoassays for detection of cryptococcal antigen in serum and cerebrospinal fluid. Clin. Vaccine Immunol. 20, 52-55. doi: 10.1128/CVI.00536-12 
Harris, J. R., Lockhart, S. R., Debess, E., Marsden-Haug, N., Goldoft, M., Wohrle, R., et al. (2011). Cryptococcus gattii in the United States: clinical aspects of infection with an emerging pathogen. Clin. Infect. Dis. 53, 1188-1195. doi: $10.1093 / \mathrm{cid} / \mathrm{cir} 723$

Hawkins, C., and Armstrong, D. (1984). Fungal infections in the immunocompromised host. Clin. Haematol. 13, 599-630.

Hawkins, C., and Flaherty, J. P. (2007). Cryptococcal osteomyelitis of the finger complicated by meningitis. Infect. Dis. Clin. Pract. 15, 345-347. doi: 10.1097/IPC.0b013e3180315170

Heenan, P. J., and Dawkins, R. L. (1981). Cryptococcosis with multiple squamous cell tumors associated with a T-cell defect. Cancer 47, 291-295.

Houda, B., Wafa, A., Zoubida, T. M., Mohamed, A., Mohamed, A., and Hicham, H. (2011). Vertebrae cryptococcosis in an immunocompetent patient-a case report. Pan. Afr. Med. J. 8, 42. doi: 10.4314/pamj.v8i1. 71158

Hummel, H., Stosiek, P., Lindner, R., and Bär, W. (1996). Osteomyelitis from Cryptococcus neoformans. Mycoses 39, 94-96. doi: 10.1111/j.14390507.1996.tb00512.x

Italiano, A., Yen, B. C., Rosenthal, S. A., and Rafii, M. (2001). Cryptococcal osteomyelitis with septic arthritis. Orthopedics 24, 59-60.

Jacobson, M. E., Griesser, M. J., Paloski, M. D., and Mayerson, J. L. (2012). Isolated Cryptococcus neoformans osteomyelitis of the proximal femur: a case report and review of literature. Orthop. Surg. 4, 190-193. doi: 10.1111/j.17577861.2012.00188.x

Jain, D., Najjar, M., Azher, Q., and Bachuwa, G. (2013). Cryptococcal sternal osteomyelitis in a healthy woman: a review of Cryptococcus neoformans. BM] Case Rep. bcr2013009129. doi: 10.1136/bcr-2013-009129

Jain, K., Mruthyunjaya, and Ravishankar, R. (2011). Cryptococcal abscess and osteomyelitis of the proximal phalanx of the hand. Indian J. Pathol. Microbiol. 54, 216-218. doi: 10.4103/0377-4929.77417

Jain, M., Sharma, S., and Jain, T. S. (1999). Cryptococcosis of thoracic vertebra simulating tuberculosis: diagnosis by fine-needle aspiration biopsy cytology-a case report. Diagn. Cytopathol. 20, 385-386.

Jou, H. J., Lee, F. T., Wang, M. N., Yang, P. Y., and Wang, N. P. (2011). Bifocal cryptococcal osteomyelitis: management of a patient with concurrent femur and rib infections. Hip. Int. 21, 495-497. doi: 10.5301/HIP.2011. 8579

Klein, K. R., Hall, L., Deml, S. M., Rysavy, J. M., Wohlfiel, S. L., and Wengenack, N. L. (2009). Identification of Cryptococcus gattii by use of L-canavanine glycine bromothymol blue medium and DNA sequencing. J. Clin. Microbiol. 47, 3669-3672. doi: 10.1128/JCM.01072-09

Kontoyiannis, D. P., Peitsch, W. K., Reddy, B. T., Whimbey, E. E., Han, X. Y., Bodey, G. P., et al. (2001). Cryptococcosis in patients with cancer. Clin. Infect. Dis. 32, E145-E150. doi: 10.1086/320524

Kromminga, R., Staib, F., Thalmann, U., Jautzke, G., Seibold, M., Hochrein, H., et al. (1990). Osteomyelitis due to Cryptococcus neoformans in advanced age. Case report and review of literature. Mycoses 33, 157-166.

Kumlin, U., Elmqvist, L. G., Granlund, M., Olsen, B., and Tärnvik, A. (1997). CD4 Lymphopeniaina patient with cryptococcal osteomyelitis. Scand. J. Infect. Dis. 29, 205-206. doi: 10.3109/00365549709035888

Levine, A. M., Meier, P., and Dorfman, H. D. (1985). Isolated cryptococcus osteomyelitis of the humerus simulating a neoplasm of bone in a patient with sarcoidosis. Case report 329. Skeletal Radiol. 14, 152-156. doi: 10.1007/BF00349754

Lie, K. W., Yu, Y. L., Cheng, I. K., Woo, E., and Wong, W. T. (1989). Cryptococcal infection of the lumbar spine. J. R. Soc. Med. 82, 172-173.

Lindsley, M. D., Mekha, N., Baggett, H. C., Surinthong, Y., Autthateinchai, R., Sawatwong, P., et al. (2011). Evaluation of a newly developed lateral flow immunoassay for the diagnosis of cryptococcosis. Clin. Infect. Dis. 53, 321-325. doi: $10.1093 / \mathrm{cid} / \mathrm{cir} 379$

Liu, P. Y. (1998). Cryptococcal osteomyelitis: case report and review. Diagn. Microbiol. Infect. Dis. 30, 33-35. doi: 10.1016/S0732-8893(97)00190-9

Magid, D., and Smith, B. (1992). Cryptococcus neoformans osteomyelitis of the clavicle. Orthopedics 15, 1068-1070.

Matsushita, T., and Suzuki, K. (1985). Spastic paraparesis due to cryptococcal osteomyelitis. A case report. Clin. Orthop. Relat. Res. 196, 279-284.

Mauri, M., Catells, L., Ruiz, I., Vargas, V., and Guardia, J. (1997). Cryptococcal arthritis as the primary form of human immunodeficiency syndrome. Enferm. Infecc. Microbiol. Clin. 15, 560.
McGuire, D. T., Bick, S. M., and Westgarth-Taylor, T. (2011). Cryptococcoma of the ilium in an immuno-competent child- a case report and review of the literature. SA. Orthop. J. 10, 74-77.

McTaggart, L., Richardson, S. E., Seah, C., Hoang, L., Fothergill, A., and Zhang, S. X. (2011). Rapid identification of Cryptococcus neoformans var. grubii, C. neoformans var. neoformans, and C. Gattii by use of rapid biochemical tests, differential media, and DNA sequencing. J. Clin. Microbiol. 49, 2522-2527. doi: 10.1128/JCM.00502-11

Meredith, H. C., John, J. F. Jr., Rogers, C. I., Gooneratne, N., and Kreutner, A. Jr. (1979). Case report 89. Skeletal Radiol. 4, 53-55. doi: 10.1007/BF00350599

Mitchell, T. G., and Perfect, J. R. (1995). Cryptococcosis in the era of AIDS-100 years after the discovery of Cryptococcus neoformans. Clin. Microbiol. Rev. 8, $515-548$.

Negroni, R. (2012). Cryptococcosis. Clin. Dermatol. 30, 599-609. doi: 10.1016/j.clindermatol.2012.01.005

Noh, H. M., Kuszyk, B. S., and Fishman, E. K. (1999). Cryptococcoma of the sacrum. Skeletal Radiol. 28, 49-51. doi: 10.1007/s002560050472

Perfect, J. R., Dismukes, W. E., Dromer, F., Goldman, D. L., Graybill, J. R., Hamill, R. J., et al. (2010). Clinical practice guidelines for the management of cryptococcal disease: 2010 update by the Infectious Diseases Society of America. Clin. Infect. 50, 291-322. doi: 10.1086/649858

Perfect, J. R., Durack, D. T., and Gallis, H. A. (1983). Cryptococcemia. Medicine 62, 98-109. doi: 10.1097/00005792-198303000-00003

Pirofski, L., and Casadevall, A. (1990). Mixed staphylococcal and cryptococcal epidural abscess in a patient with AIDS. Rev. Infect. Dis. 12, 964-965. doi: 10.1093/clinids/12.5.964

Poliner, J. R., Wilkins, E. B., and Fernald, G. W. (1979). Localized osseous cryptococcosis. J. Pediatr. 94, 597-579. doi: 10.1016/S0022-3476(79)80020-7

Prendiville, S., Bielamowicz, S. A., Hawrych, A., and Deeb, Z. E. (2000). Isolated cryptococcal sphenoid sinusitis with septicemia, meningitis, and subsequent skull base osteomyelitis in an immunocompetent patient. Otolaryngol. Head Neck Surg. 123, 277-279. doi: 10.1067/mhn.2000.104777

Qadir, I., Ali, F., Malik, U. Z., and Umer, M. (2011). Isolated cryptococcal osteomyelitis in an immunocompetent patient. J. Infect. Dev. Ctries. 5, 669-673.

Raftopoulos, I., Meller, J. L., Harris, V., and Reyes, H. M. (1998). Cryptococcal rib osteomyelitis in a pediatric patient. J. Pediatr. Surg. 33, 771-773. doi: 10.1016/S0022-3468(98)90216-0

Ramkillawan, Y., Dawood, H., and Ferreira, N. (2013). Isolate cryptococcal osteomyelitis in an immune-competent patient host: a case report. Int. J. Infect. Dis. 17, e1229-e1231. doi: 10.1016/j.ijid.2013.04.013

Reinig, J. W., Hungerford, G. D., Mohrmann, M. E., and Vera, C. L. (1984). Case report 268. Diagnosis: cryptococcal osteomyelitis of the calvaria. Skeletal Radiol. 11, 221-223. doi: 10.1007/BF00349499

Ricciardi, D. D., Sepkowitz, D. V., Berkowitz, L. B., Bienenstock, H., and Maslow, M. (1986). Cryptococcal arthritis in a patient with acquired immune deficiency syndrome. Case report and review of the literature. J. Rheumatol. 13, 455-458.

Saeed, M. U., Dacuycuy, M. A., and Kennedy, D. J. (2009). Cryptococcal epidural abscess with bone involvement: case report and review of literature. Infect. Dis. Clin. Pract. 17, 193-195. doi: 10.1097/IPC.0b013e3181812c14

Schmidt, D. M., Sercarz, J. A., Kevorkian, K. F., and Canalis, R. F. (1995). Cryptococcosis presenting as a neck mass. Ann. Otol. Rhinol. Larygol. 104, 711-714. doi: 10.1177/000348949510400908

Shaff, M. I., Berger, J. L., and Green, N. E. (1982). Cryptococcal osteomyelitis, pulmonary sarcoidosis, and tuberculosis in a single patient. South. Med. J. 75, 225-226. doi: 10.1097/00007611-198202000-00026

Singh, N., Rihs, J., Gaywoski, T., and Yu, V. (1994). Cutaneous cryptococcosis mimicking bacterial cellulitis in a liver transplant recipient: case report and review in solid organ transplant recipients. Clin. Transpl. 8, 365-368.

Singh, R., and Xess, I. (2010). Multiple osseous involvements in a case of disseminated cryptococcosis. Indian J. Orthop. 44, 336-338. doi: 10.4103/00195413.65158

Sinnott, J. T., and Holt, D. A. (1989). Cryptococcal pyarthrosis complicating gouty arthritis. South. Med. J. 82, 1555-1556. doi: 10.1097/00007611-19891200000024

Sorensen, R. U., Boehm, K. D., Kaplan, D., and Berger, M. (1992). Cryptococcal osteomyelitis and cellular immunodeficiency associated with interleukin-2 deficiency. J. Pediatr. 121, 873-879. doi: 10.1016/S0022-3476(05) 80331-2 
Speed, B., and Dunt, D. (1995). Clinical and host differences between infections with the two varieties of Cryptococcus neoformans. Clin. Infect. Dis. 21, 28-34. doi: 10.1093/clinids/21.1.28

Stead, K. J., Klugman, K. P., Painter, M. L., and Koornhof, H. J. (1988). Septic arthritis due to Cryptococcus neoformans. J. Infect. 17, 139-145. doi: 10.1016/S01634453(88)91683-0

Thompson, G. R., Wiederhold, N. P., Fothergill, A. W., Vallor, A. C., Wickes, B. L., and Patterson, T. F. (2009). Antifungal susceptibilities among different serotypes of Cryptococcus gattii and Cryptococcus neoformans. Antimicrob. Agents. Chemother. 53, 309-311. doi: 10.1128/AAC. 01216-08

Ueda, Y., Roessner, A., Edel, G., Wuisman, P., Erlemann, R., and Böcker, W. (1992). Case report 699. Primary localized skeletal cryptococcosis (torulosis) of the right tibia at its proximal end. Skeletal Radiol. 21, 117-119.

Venkatachala, S., Naik, D. R., Shanthakumari, S., and Jayaraman, A. (2010) Acquired immunodeficiency syndrome presenting as marrow cryptococcosis. Indian J. Pathol. Microbiol. 53, 904-906. doi: 10.4103/0377-4929. 72069

Wildstein, M. S., Martin, S. M., and Glaser, J. A. (2005). Cryptococcal osteomyelitis in a 20-year-old male with sarcoidosis. Spine J. 5, 467-470. doi: 10.1016/j.spinee.2004.12.002

Witte, D. A., Chen, I., Brady, J., Ramzy, I., Truong, L. D., and Ostrowski, M. L. (2000). Cryptococcal osteomyelitis. Report of a case with aspiration biopsy of a humeral lesion with radiologic features of malignancy. Acta Cytol. 44, 815-818. doi: $10.1159 / 000328567$

Wood, L., and Miedzinski, L. (1996). Skeletal cryptococcosis: case report and review of the literature. Can. J. Infect. Dis. 7, 125-132.

Yu, J. Q., Tang, K. J., Xu, B. L., Xie, C. M., and Light, R. W. (2012). Pulmonary cryptococcosis in non-AIDS patients. Infect. Dis. 16, 531-539. doi: 10.1016/j. bjid.2012.07.004
Zach, T. L., and Penn, R. G. (1986). Localized cryptococcal osteomyelitis in an immunocompetent host. Pediatr. Infect. Dis. 5, 601-603. doi: 10.1097/00006454-198609000-00029

Zanelli, G., Sansoni, A., Ricciardi, B., Ciacci, C., and Cellesi, C. (2001). Muscularskeletal cryptococcosis in a patient with idiopathic CD4+ lymphopenia. Mycopathologia 149, 137-139. doi: 10.1023/A:1007271822625

Zhang, Y., Yu, Y. S., Tang, Z. H., and Zang, G. Q. (2012). Cryptococcal osteomyelitis of the scapula and rib in an immunocompetent patient. Med. Mycol. 50, 751-755. doi: 10.3109/13693786.2012.670277

Zhou, H. X., Ning, G. Z., Feng, S. Q., Jia, H. W., Liu, Y., Feng, H. Y., et al. (2013) Cryptococcosis of lumbar vertebra in a patient with rheumatoid arthritis and scleroderma: case report and literature review. BMC Infect. Dis. 13:128. doi: $10.1186 / 1471-2334-13-128$

Conflict of Interest Statement: The authors declare that the research was conducted in the absence of any commercial or financial relationships that could be construed as a potential conflict of interest.

Received: 29 August 2014; accepted: 05 December 2014; published online: 14 January 2015.

Citation: Zhou H-X, Lu L, Chu T, Wang T, Cao D, Li F, Ning G and Feng S (2015) Skeletal cryptococcosis from 1977 to 2013. Front. Microbiol. 5:740. doi: 10.3389/fmicb. 2014.00740

This article was submitted to Infectious Diseases, a section of the journal Frontiers in Microbiology.

Copyright (C) $2015 \mathrm{Zhou}, \mathrm{Lu}$, Chu, Wang, Cao, Li, Ning and Feng. This is an openaccess article distributed under the terms of the Creative Commons Attribution License (CC BY). The use, distribution or reproduction in other forums is permitted, provided the original author(s) or licensor are credited and that the original publication in this journal is cited, in accordance with accepted academic practice. No use, distribution or reproduction is permitted which does not comply with these terms. 